\title{
Pharmacological Interventions Including Medical Injections for Neck Pain: An Overview as Part of the $\mathrm{ICON}^{\S}$ Project
}

\author{
Paul M. Peloso ${ }^{1}$, Mahweesh Khan ${ }^{2}$, Anita R. Gross ${ }^{*}, 2$, Lisa Carlesso², Lina Santaguida ${ }^{2}$, \\ Janet Lowcock ${ }^{2}$, Joy C MacDermid ${ }^{2}$, Dave Walton ${ }^{3}$, Charlie H. Goldsmith ${ }^{4}$, \\ Pierre Langevin ${ }^{5}$ and Qiyun $\mathrm{Shi}^{3}$
}

\author{
${ }^{I}$ Merck \& Co., Rahway, NJ, USA \\ ${ }^{2}$ McMaster University, Hamilton, Canada \\ ${ }^{3}$ Western University, London, Canada \\ ${ }^{4}$ Simon Fraser University, Vancouver, Canada \\ ${ }^{5}$ Laval University, Quebec City, Canada
}

\begin{abstract}
Objectives: To conduct an overview (review-of-reviews) on pharmacological interventions for neck pain.
Search Strategy: Computerized databases and grey literature were searched from 2006 to 2012.

Selection Criteria: Systematic reviews of randomized controlled trials (RCT) in adults with acute to chronic neck pain reporting effects of pharmacological interventions including injections on pain, function/disability, global perceived effect, quality of life and patient satisfaction.

Data Collection \& Analysis: Two independent authors selected articles, assessed risk of bias and extracted data The GRADE tool was used to evaluate the body of evidence and an external panel provided critical review.

Main Results: We found 26 reviews reporting on 47 RCTs. Most pharmacological interventions had low to very low quality methodologic evidence with three exceptions. For chronic neck pain, there was evidence of:

1) a small immediate benefit for eperison hydrochloride (moderate GRADE, 1 trial, 157 participants);

2) no short-term pain relieving benefit for botulinum toxin-A compared to saline (strong GRADE; 5 trial meta-analysis, 258 participants) nor for subacute/chronic whiplash (moderate GRADE; 4 trial meta-analysis, 183 participants) including reduced pain, disability or global perceived effect; and

3) no long-term benefit for medial branch block of facet joints with steroids (moderate GRADE; 1 trial, 120 participants) over placebo to reduce pain or disability;

Reviewers' Conclusions: While in general there is a lack of evidence for most pharmacological interventions, current evidence is against botulinum toxin-A for chronic neck pain or subacute/chronic whiplash; against medial branch block with steroids for chronic facet joint pain; but in favour of the muscle relaxant eperison hydrochloride for chronic neck pain.
\end{abstract}

Keywords: Neck pain, pharmacological interventions, medical injections, review of reviews.

\section{INTRODUCTION}

Neck pain is common, is experienced by approximately one third of adults over the course of one year [1], can be

*Address correspondence to this author at the McMaster University, School of Rehabilitation Science, IAHS, 1400 Main Street West, $4^{\text {th }}$ floor, Hamilton, ON, L8S 1C7, Canada; Tel: 905-577-0098; Fax: 905-577-0603; E-mail: grossa@mcmaster.ca

${ }^{\S}$ ICON (International Collaboration on Neck)

Expert Panel: Helge Kasch, MD - Neurology, Aarhus University Hospital, Aarhus, Denmark; Jan Hartvigsen, DC, PhD, University of Southern Denmark; Odense M, Denmark; Marc White, MD, PhD - Family Medicine, Massage Therapy, Canadian Institute for the Relief of Pain and Disability, Vancouver, Canada; Margareta Nordin, DMedSci, New York University Hospital for Joint Diseases, New York, USA; Justin Kenardy, Clinical Health Psychologist, PhD, The University of Queensland, Royal Brisbane and Women's Hospital, QLD, Australia; Norm Buckley, MD - Anesthesia, McMaster University, Hamilton, Canada severely disabling and is contributing to rising socio-economic costs and societal burden [2]. Patients most commonly seek care from medical doctors, and physicians typically prescribe pharmacological interventions [3]. A variety of medications and medicinal injections are used to reduce transient, recurrent or persisting neck pain and disability in the acute or chronic stages of the disorder. Physicians may chose from various classes of medications (see APPENDIX 1) including: nonopioid analgesics, oral and topical NSAIDs, opioids, muscle relaxants, benzodiazepines, tricyclic antidepressants and GABA derivatives. Medicinal injections might also be used including: corticosteroids, anesthetics, and neuromuscular paralytic agent (botulinum toxins).

The choice of a specific agent often considers the mechanism of action of the specific drug [4-6], it presumed efficacy and adverse events, the individual patient, including past therapies tried, and should also be informed by evidence 
that the chosen intervention will lead to the therapeutic objective in that patient population. For non-opioid analgesics like acetaminophen (e.g. Tylenol ${ }^{\circledR}$ ) the mechanism of action remains unclear but may include inhibition of cyclooxygenase (COX) enzymes; it typically well tolerated with limited adverse effects. NSAIDs act by blocking cyclo-oxygenase (COX) enzymes 1 \& 2. It's thought that blocking COX-2 decreases pain and inflammation while also reducing the risk of gastrointestinal adverse effects (ulcers, bleeding) that result from COX-1 inhibition on the GI mucosa and platelets, whereas controversy remains over the role of NSAIDs and cardiovascular adverse effects [4]. Common oral NSAIDS include ibuprofen (e.g. $\operatorname{advil}^{\circledR}, \operatorname{motrin}^{\circledR}$ ), naproxen (e.g. aleve $^{\circledR}, \quad$ naprosyn $\left.^{\circledR}\right)$, diclofenac (e.g. voltaren ${ }^{\circledR}$ ) with celecoxib (celebrex ${ }^{\circledR}$ ) an example of a COX-1 sparing NSAID. Opioid medications' analgesia is obtained principally through mu-opioid receptors, with opioids most often used for chronic pain refractory to other therapies. Opioids can produce respiratory depression, nausea, vomiting, dizziness and addictive behavior in susceptible individuals. Societal concerns of diversion also limit their use. Tricyclic antidepressants increase serotonin and norepinephrine and commonly produce drowsiness, dry mouth, blurred vision, constipation, urinary retention and weight gain. Selective serotonin and norepinephrine reuptake inhibitors (e.g. duloxetine, venlafaxine) antidepressants increase serotonin and norepinephrine and have common side effects of nausea and dizziness. Anticonvulsants such as gabapentin or pregabalin decrease excitatory neurotransmitters such as glutamate and their side effects commonly include drowsiness, dizziness, unsteadiness and unclear thinking. Topical anesthetics such as lidocaine block sodium channels and are well tolerated as a 5\% topical gel. Injections include botulinum toxins and specifically Type A that are used for the treatment of muscle pain disorders and act presynaptically through inhibition of acetylcholine synthesis or its release. This blocks neuromuscular transmission at the neuromuscular junction, causing paralysis of the injected skeletal muscle. It's presumed that injecting an overactive muscle will decrease its level of contraction and allow improved reciprocal motion, improving movement and the ability to exercise. Side effects are generally minor and temporary but rare allergic reactions can occur. Finally, corticosteroid injections are administered intraarticularly and intramuscularly with the thought that they reduce inflammation (pain and swelling) at the injury site. Short term problems with injections include flushing, transient hypertension, and serum glucose fluctuation. Chronic corticosteroid use can lead to hypergycemia, insulin resistance, hypertension, weight gain, osteoporosis, anxiety, depression and cataracts.

In a previous 2009 overview, we found an insufficient evidence base on the benefits and risks of most pharmacological interventions for neck pain including the commonly used injections, which limits the ability to provide strong clinical guidance on appropriate use [7]. We found limited evidence supporting methylprednisone for acute whiplash, intramuscular lidocaine for chronic neck pain and epidural methylprednisone and lidocaine for chronic neck pain. We recommended against botulinum
toxin-A as it was not found superior to saline for chronic neck pain.

There have been further clinical trials in neck pain patients since our 2009 review and since neck pain continues to be a common potentially disabling clinical condition, we wanted to update the evidence on oral, topical and injected medications for neck pain. The purpose of this overview is to systematically review existing reviews of randomized controlled trials published after 2006 and to consider establishing evidence-based recommendations on medicines and medicinal injections for neck pain, regardless of pain duration across several diagnostic groups (e.g. including non-specific and specific neck pain with and without cervicogenic headache, radiculopathy or associated whiplash injury), while also considering varying duration of study patient follow-up (short- and long-term) and differing control groups (placebo control or active treatment) in these trials. Clinical trial outcomes we considered of primary interest were pain, function, disability, work related function, patient satisfaction, global perceived effect and quality of life as well as adverse effects of these medicines.

\section{METHODS}

Our systematic overview process included comprehensive computerized search strategies from January 2000 to August 2010: MEDLINE, EMBASE, CINAHL, ILC, CENTRAL, and LILACS, with selection criteria listed in Table $\mathbf{1}$ and at least 2 independent reviewers selecting articles, performing methodological quality assessment using the AMSTAR tool [8] qualitative assessment of the strength of evidence. We used a team consensus approach to qualitatively assess the totality of the evidence, using the Grading of Recommendations Assessment, Development and Evaluation (GRADE) methodology [9]. Further details on the methodological approach are provided in our ICON methods report [10] including search terms and strategies. Two separate searches were performed, one for treatment benefits and one for harms. The protocol for this overview was not registered.

We supplemented our computerized search strategy by identifying on-going systematic reviews in the grey literature nearing completion (e.g. Cochrane Reviews) up to July 2012 , by asking our expert panel to identify ongoing reviews and by scrutinizing the reference lists of all of the primary studies.

Data extraction was performed using forms we piloted first by one reviewer and then checked by a second. Disagreements were resolved through review of data extraction forms, discussion and consensus. We systematically extracted data from selected systematic reviews and produced evidence tables. Key factors extracted from the original reviews included the following three items: 1) descriptive features of the original review (e.g. authors, publication year, disorder, symptom duration, intervention, and comparator used such as placebo, no care, usual care, and other treatment), as well as noting the authors of primary studies included in the review; 2) methodological details of the original review (e.g. search period, AMSTAR score, quality ranking system, evidence statement and final GRADE). If the original review did not report using GRADE methodology, an estimate for GRADE was made by us based 
Table 1. Inclusion and Exclusion Criteria Set a Priori

\begin{tabular}{|c|l|}
\hline PICOSS & \multicolumn{1}{|c|}{ Criteria } \\
\hline \hline Participant & $\begin{array}{l}\text { Adult ( } \geq 18 \text { years), acute to chronic non-specific or specific neck pain with or without cervicogenic headache or radiculopathy or } \\
\text { whiplash associated disorders (WAD) }\end{array}$ \\
\hline Intervention & $\begin{array}{l}\text { Pharmacological interventions including medical injections; } \\
\text { Exclusion: Alternative medicines such as homeopathy, herbal medicines, naturopathic medicines }\end{array}$ \\
\hline Comparison & Placebo control or comparison (i.e. standard care, another treatment) \\
\hline Outcomes & $\begin{array}{l}\text { Primary: pain, function, disability, work related, quality of life } \\
\text { Secondary: global perceived effect and patient satisfaction }\end{array}$ \\
\hline Study Design & Systematic reviews of randomized trials; Exclusion: narrative reviews were excluded \\
\hline Study Timeframe & $\begin{array}{l}\text { Immediate post-treatment (IP), short-term (ST: closest to 3 months); intermediate-term (IT: closest to 6 months); long-term (LT: } \\
\text { closest to 1 year) }\end{array}$ \\
\hline
\end{tabular}

on the methodological details in the systematic review; and 3) data on benefits and risks (e.g. effect size, effect direction, duration of follow-up, reports of harm). Summary statements on harms included information reported in the original reviews as well as information obtained from the second literature search on harms.

We employed the following a priori triage rules to facilitate decisions on including/excluding reviews:

1) Type of treatment (analgesic, NSAID, opioid, etc.) reviews by drug class. See APPENDIX 1 for a complete list of medications and injections considered by medicine treatment category.

2) Within a treatment drug class we grouped data by type of comparator (placebo, active treatments, etc.).

3) We prioritized the highest quality reviews based on the rules below, PER grouping.

a. If there were few reviews, we retained them all.

b. If there were several reviews reporting on the same treatments and comparators, we retained the highest quality reviews, using the approach recommended by Whitlock [11]. Whitlock has suggested considering the following factors: i. Year of publication. We selected the most recent reviews when the data was similar across reviews and there was no loss of studies contained in the older reviews. We further ensured consistency among reviews' conclusions before eliminating older reviews. Inconsistency and discordance were highlighted and potential reasons for differences were discussed; ii. AMSTAR risk of bias. We prioritized reviews with a low risk of bias. Reviews that scored 8 or higher on the 11-point AMSTAR scale were considered at low risk of bias; moderate risk of bias was considered for scores between 5 and 7; and a high risk of bias was assigned to scores of 4 or less. These various reviews were then further summarized in a "Summary of Findings" table to facilitate incorporating this information into clinical practice. Inconsistency and discordance were highlighted and discussed across reviews; iii. Effect size estimates: We considered effect sizes as the primary summary measure. Within our defined groups of treatments and comparators, we selected a review that best represented the treatment effect sizes (including through meta-analysis) although we also report the range of estimates from the other included reviews. In cases where there was a large discordance between reviews, we reported our own analysis using the individual studies included in the reviews. Additional data on magnitude of effects such as number-needed-to-treat (NNT) and weighted mean difference (WMD) were also extracted when possible. Further we also considered the clinical importance of these effects using several guiding principles. We considered the published data on the minimal detectable change and the minimal clinically important difference for that outcome. We used a change from baseline of $\geq 15 \%$ to represent the MCID when it was not otherwise published. We also considered the magnitude of the treatment effect (represented by WMD, NNT, absolute benefit, treatment advantage), the evidence for a dose-response gradient, and evidence on the duration of effect (See APPENDIX 2) [12-15] in our assessment of clinical relevance.

4) Strength of Evidence using GRADE approach: We considered the same prioritized systematic review to represent the body of evidence for any treatment and assigned an overall GRADE on the strength of evidence. If the selected (prioritized) reviews already reported a GRADE table, we used that. As a reminder, the GRADE approach assessing the quality of evidence from primary trials considers information on design (randomized controlled trials or RCT), information on timing of outcomes (immediately post treatment or IP to long term or LT follow-up); risk of bias; imprecision based on sample size; inconsistency across trials; indirectness and reporting bias. 


\section{RESULTS}

From 10, 055 reviews that were screened and 117 eligible reviews relating to neck pain filters and adverse events filters, 43 reviews related to medicines were ultimately considered for inclusion in this report. A total of 26 treatment reviews and 6 harm related reviews were included for this topic (see Fig. 1 - PRISMA diagram [16]). Excluded reviews are presented in Appendix 3, along with an accompanying rationale for their exclusion. Using the selected (prioritized) reviews, we report on trial findings by "overall quality of evidence" using the GRADE approach and stratified by the pre-determined treatment category in the Summary of Findings table (Table 2) [17-90]. We report on conflicting evidence across reviews in Table $\mathbf{3}$ [91, 92]. Our final recommendations are summarized in the Evidencebased Recommendation table and provided in Table 4. The AMSTAR assessment (see Table 5) revealed that the most common methodological limitations among the included reviews were incomplete reporting on: publication bias; conflict of interest; and full reporting of excluded studies.
Details on risk of bias (AMSTAR scoring) are available in the companion methods paper by Santaguida et al. APPENDIX 2 to this report provides the details on why 17 medicines reviews were excluded. Table $\mathbf{2}$ provides summary findings by treatment category and includes the primary trials and related systematic review(s) used to compile recommendations in this systematic review. The evidence tables, 'Characteristics of Included Studies and GRADE rationale', underpinning the summary provided in Table 2 are available from the authors. Table 6 [93-98] summarizes the findings on harms. The primary reviews included in our analyses considered the following medicines and medical injection therapies: anti-inflammatories and analgesics in combination, anti-inflammatories alone, analgesics alone, anesthetics such as lidocaine intramuscular (IM and topical nerve blocks), muscle relaxants, neurotropic multivitamins (IM), psychotropic agents, sterile water (IM, subcutaneous and intracutaneous), subcutaneous insufflation, botulinum toxin-A (IM), and corticosteroids (intra-articular, intravenous, epidural).
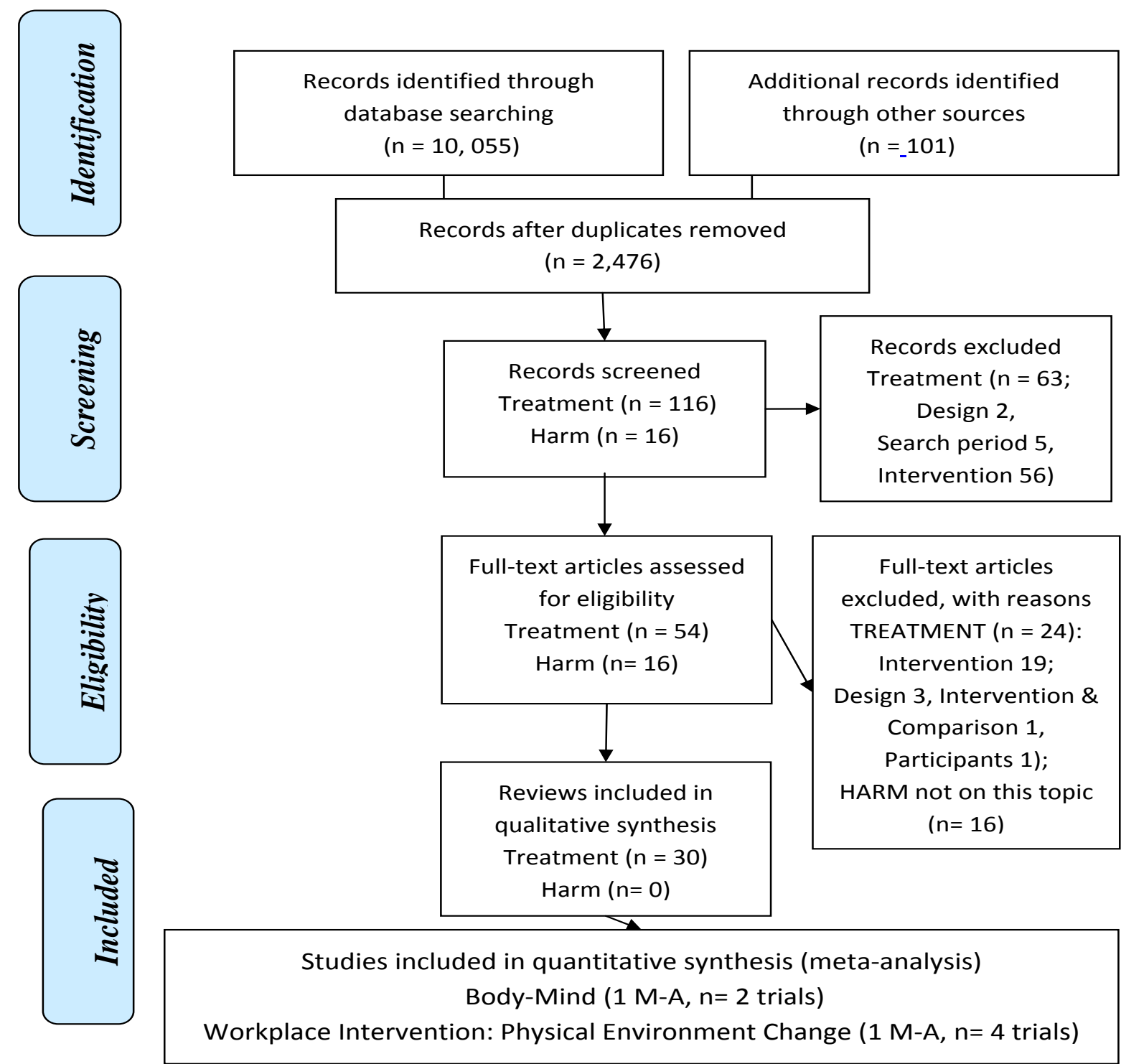

Fig. (1). PRISMA diagram showing the flow of reviews. 
Table 2. Summary of Findings by GRADE (Quality of Evidence)

\begin{tabular}{|c|c|c|c|c|c|c|}
\hline \multirow[t]{2}{*}{ Category } & \multirow{2}{*}{$\begin{array}{c}\text { Treatments Details } \\
\text { Disorder Characteristic }\end{array}$} & \multirow{2}{*}{$\begin{array}{c}\text { vs Comparison } \\
\text { Primary Authors } \\
\text { (REVIEW Reference) }\end{array}$} & \multicolumn{4}{|c|}{ Quality of Evidence (GRADE*) } \\
\hline & & & Strong & Moderate & Low & Very Low \\
\hline \multicolumn{7}{|c|}{ EVIDENCE of BENEFIT - Medical Injections and Oral Medication } \\
\hline 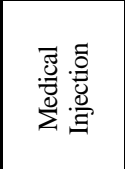 & $\begin{array}{l}\text { Intravenous Glucocorticoid } \\
\text { for acute WAD }\end{array}$ & $\begin{array}{l}\text { vs placebo } \\
\text { Petterson } 1998 \text { [17] } \\
\text { (PELOSO 2007 [7]; } \\
\text { CONLIN 2005 [18]) }\end{array}$ & & & $\begin{array}{l}\text { IT sick leave } \\
\text { IT pain (neg) }\end{array}$ & \\
\hline 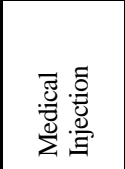 & $\begin{array}{l}\text { Intramuscular injection lidocaine + } \\
\text { stretch } \\
\text { for chronic MND (myofascial pain) }\end{array}$ & $\begin{array}{l}\text { vs saline + stretch } \\
\text { Esenyel 2000 [19] } \\
\text { (PELOSO 2007 [7]; } \\
\text { GROSS 2007 [20]) }\end{array}$ & & & & ST pain \\
\hline 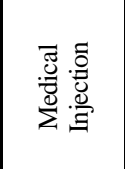 & $\begin{array}{l}\text { Intramuscular injection lidocaine } \\
\text { for chronic non-specific mechanical } \\
\text { neck pain }\end{array}$ & $\begin{array}{l}s \text { dry needling } \\
\text { Hong } 1994 \text { [21] } \\
\text { (PELOSO 2007 [7]; } \\
\text { TSAKITZIDIS 2009 [22]) }\end{array}$ & & & ST pain & \\
\hline 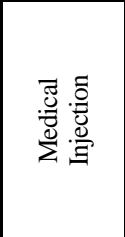 & $\begin{array}{l}\text { tramuscular injection Botulinium A } \\
+ \text { exercise /medication } \\
\text { for subacute/chronic WAD and } \\
\text { non-specific neck pain }\end{array}$ & $\begin{array}{l}\text { vs saline }+ \\
\text { exercise/medication } \\
\text { Braker } \text { et al. } 2008 \text { [23]; } \\
\text { Lew } \text { et al. } 2008 \text { [24]; } \\
\text { Ferrante } \text { et al. } 2005 \text { [25] } \\
\text { (LANGEVIN } 2011 \text { [26]) }\end{array}$ & & & & ST pain \\
\hline 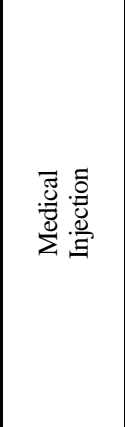 & $\begin{array}{l}\text { Epidural steroid injection }+/- \\
\text { lidocaine } \\
\text { for a), b) chronic neck pain with } \\
\text { radiculopathy } \\
\text { c) chronic neck pain with } \\
\text { radiation }\end{array}$ & $\begin{array}{l}\text { vs intramuscular injection } \\
\text { steroid and lidocaine } \\
\text { a) Stav et al. } 1993 \text { [27], } \\
\text { b) Castagnara } \text { et al. } 1994 \\
\text { [28], } \\
\text { vs continuous epidural } \\
\text { c) Pasqualucci } \text { et al. } 2007 \\
\text { [29] } \\
\text { (BENYANMIN 2009 [30]; } \\
\text { PELOSO 2007 [7]; ABDI } \\
\text { 2007 [31], ABDI 2005 } \\
\text { [32] ) }\end{array}$ & & & $\begin{array}{l}\text { a) LT pain, } \mathrm{LT} \\
\text { return to work, LT } \\
\text { range of } \\
\text { movement } \\
\text { b) LT pain } \\
\text { c) IT pain, IT sleep }\end{array}$ & \\
\hline 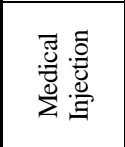 & $\begin{array}{l}\text { Subcutaneious sterile water injection } \\
\text { for chronic neck pain after whiplash }\end{array}$ & $\begin{array}{l}\text { vs placebo } \\
\text { Bryn } \text { et al. } 1993[37] \\
\text { (TEASELL } 2010[38,39]\end{array}$ & & & & ST pain \\
\hline 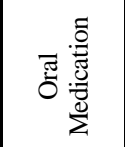 & $\begin{array}{l}\text { Cyclobenzaprine (psychotropic } \\
\text { agent) + Lysinine Cloniximate } \\
\text { (NSAID) } \\
\text { for subacute MND }\end{array}$ & $\begin{array}{l}\text { vs lysinine cloniximate } \\
\text { Nasswetter et al. 1998 [40] } \\
\text { (PELOSO 2007 [7]) }\end{array}$ & & & & $\begin{array}{l}\text { IP pain } \\
\text { ST pain }\end{array}$ \\
\hline 苛: & $\begin{array}{l}\text { Tetrazepam (psychotropic agent) }+ \\
\text { Paracetamol (analgesic) } \\
\text { for acute MND }\end{array}$ & $\begin{array}{l}\text { vs paracetamol } \\
\text { Salzmann et al. } 1993 \text { [41] } \\
\text { (PELOSO 2007 [7]) }\end{array}$ & & & & IP pain IP ROM IP GPE \\
\hline चू & $\begin{array}{l}\text { Eperison Hydrochloride } \\
\text { (psychotropic agent) } \\
\text { for chronic MND }\end{array}$ & $\begin{array}{l}\text { vs placebo } \\
\text { Bose at al } 1999[42] \\
\text { (PELOSO 2007 [7]) }\end{array}$ & & $\begin{array}{l}\text { IP pain } \\
\text { IP ROM }\end{array}$ & & \\
\hline 팸: & $\begin{array}{l}\text { Chlormezanone (muscle relaxant) } \\
\text { for subacute non specific neck pain }\end{array}$ & $\begin{array}{l}\text { vs placebo } \\
\text { Berry et al. } 1981 \text { [43] } \\
\text { (HURWITZ 2008 [44]) }\end{array}$ & & & & IP sleep \\
\hline
\end{tabular}


(Table 2) contd......

\begin{tabular}{|c|c|c|c|c|c|c|}
\hline \multirow[t]{2}{*}{ Category } & \multirow{2}{*}{$\begin{array}{c}\text { Treatments Details } \\
\text { Disorder Characteristic }\end{array}$} & \multirow{2}{*}{$\begin{array}{c}\text { vs Comparison } \\
\text { Primary Authors } \\
\text { (REVIEW Reference) }\end{array}$} & \multicolumn{4}{|c|}{ Quality of Evidence (GRADE*) } \\
\hline & & & Strong & Moderate & Low & Very Low \\
\hline \multicolumn{6}{|c|}{ EVIDENCE of BENEFIT - Medical Injections and Oral Medication } & \\
\hline 苛 & $\begin{array}{l}\text { Piroxicam (anti-inflammatory) } \\
\text { for chronic non specific neck pain } \\
\text { (Note: cervicobrachial pain - went to } \\
\text { original article) }\end{array}$ & \begin{tabular}{|l|} 
vs placebo \\
Yamamoto et al. 1983 [45] \\
(HURWITZ 2008 [44])
\end{tabular} & & & $\begin{array}{l}\text { ST pain,physician } \\
\text { perceived } \\
\text { improvement }\end{array}$ & \\
\hline 急 & $\begin{array}{l}\text { Indomethacin (anti-inflammatory) } \\
\text { for non specific neck pain }\end{array}$ & \begin{tabular}{|l|} 
vs placebo \\
Yamamoto et al. 1983 [45] \\
(HURWITZ 2008 [44])
\end{tabular} & & & $\begin{array}{l}\text { ST pain, physician } \\
\text { perceived } \\
\text { improvement }\end{array}$ & \\
\hline 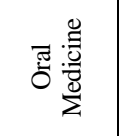 & $\begin{array}{l}\text { Tolmetin (anti-inflammatory) } \\
\text { for MND, Osteoarthritis }\end{array}$ & $\begin{array}{l}\text { vs naproxen } \\
\text { Ginsbert et al. } 1980 \text { [46] } \\
\text { (PELOSO 2007 [7]) }\end{array}$ & & & $\begin{array}{l}\text { IP pain } \\
\text { IP ROM }\end{array}$ & \\
\hline 可 & $\begin{array}{l}\text { Benorylate (analgesic) } \\
\text { for subacute to chronic non specific } \\
\text { neck pain } \\
\text { (Note: across } 6 \text { disorder types - } 90 \\
\text { patients with degenerative disease, } \\
\mathrm{n}=20 \text { with cervical spondylosis (had } \\
\text { to go to original article to retrieve } \\
\text { information) }\end{array}$ & $\begin{array}{l}\text { vs placebo } \\
\text { Berry et al. } 1981 \text { [43] } \\
\text { (HURWITZ } 2008 \text { [44]) }\end{array}$ & & & & $\begin{array}{l}\text { IP pain } \\
\text { IP stiffness } \\
\text { IP sleep } \\
\text { IP ability to work }\end{array}$ \\
\hline 可 & $\begin{array}{l}\text { Benorylate (analgesic) + } \\
\text { Chlormezanone (muscle relaxant) } \\
\text { for subacute non specific neck pain }\end{array}$ & $\begin{array}{l}\text { vs placebo } \\
\text { Berry et al. } 1981 \text { [43] } \\
\text { (HURWITZ } 2008 \text { [44]) }\end{array}$ & & & & IP pain \\
\hline तु & $\begin{array}{l}\text { Paracetamol (analgesic) + } \\
\text { Orphenadrine (anticholinergic) - } \\
\text { Norgesic } \\
\text { for non specific neck pain }\end{array}$ & $\begin{array}{l}\text { vs placebo } \\
\text { Hoivik et al. } 1983 \text { [47] } \\
\text { (HURWITZ } 2008 \text { [44]; } \\
\text { LEAVER } 2010 \text { [48]) }\end{array}$ & & & ST pain & \\
\hline 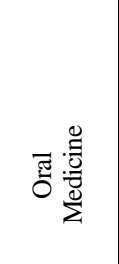 & $\begin{array}{l}\text { Oxycodone Controlled Release } \\
\text { (opioid analgesic) } \\
\text { for non specific neck pain (acute } \\
\text { chronic neck pain flares) }\end{array}$ & $\begin{array}{l}\text { vs placebo } \\
\text { Ma et al. } 2008 \text { [449 } \\
\text { (TSAKITZIDIS } 2009 \text { [22]) }\end{array}$ & & & $\begin{array}{l}\text { ST pain, frequency } \\
\text { of patients' pain } \\
\text { episodes, quality of } \\
\text { life, quality of } \\
\text { sleep }\end{array}$ & \\
\hline \multicolumn{6}{|c|}{ EVIDENCE of NO BENEFIT ( $v s$ control) or No DIFFERENCE ( $v s$ another treatment) - Medical Injections and Oral Medication } & \\
\hline 呇 & $\begin{array}{l}\text { Botulinum-A injection } \\
\text { a) for chronic MND with or without } \\
\text { radiculopathy or headache } \\
\text { b) for chronic cervicogenic headache } \\
\text { pain and disability } \\
\text { c) for chronic myofascial neck and } \\
\text { shoulder pain }\end{array}$ & $\begin{array}{l}\text { vs saline } \\
\text { a) Cheshire } \text { et al. } 1994 \\
\text { [50]; Gobel } \text { et al. } 2006 \\
\text { [51]; } \\
\text { Ojala } \text { et al. } 2006 \text { [52]; } \\
\text { Lew } \text { et al. } 2008 \text { [24] } \\
\text { (LANGEVIN } 2011 \\
\text { [26,90]) } \\
\\
\text { b) Schnider } 2002 \text { [53], } \\
\text { Freund 2000 [54] } \\
\text { (LANGEVIN 2011 } \\
\text { [26,90]) } \\
\text { c) Wheeler 2001 [55] } \\
\text { (LANGEVIN 2011 } \\
\text { [26,90]) }\end{array}$ & $\begin{array}{l}\text { a) ST pain (neg M- } \\
\text { A) }\end{array}$ & & $\begin{array}{l}\text { b) ST, IT pain } \\
\text { (neg) } \\
\text { ST disability (neg) } \\
\text { c) IT disability } \\
\text { (neg) } \\
\text { IT GPE (neg) }\end{array}$ & \\
\hline
\end{tabular}




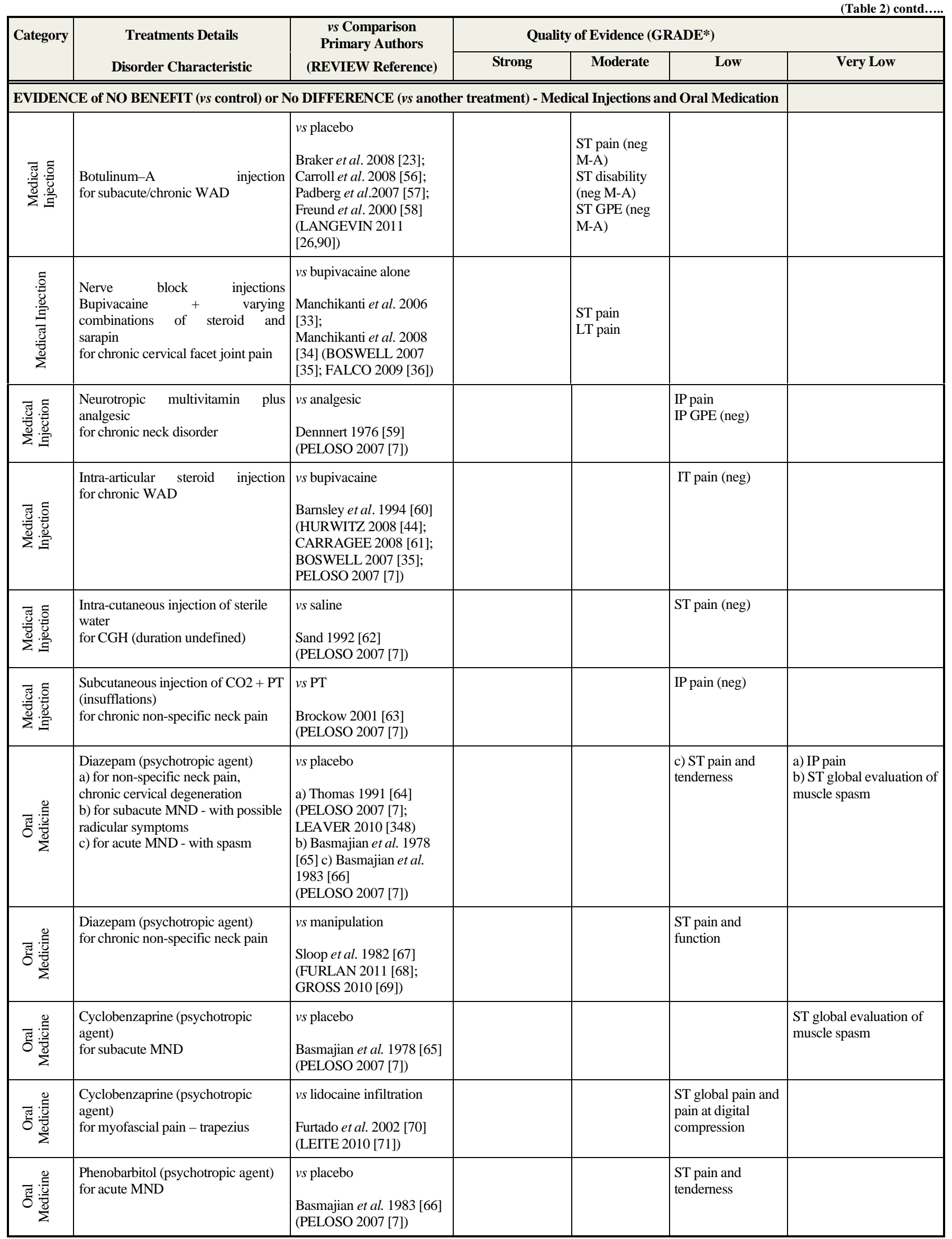




\begin{tabular}{|c|c|c|c|c|c|c|}
\hline \multirow[t]{2}{*}{ Category } & \multirow{2}{*}{$\begin{array}{c}\text { Treatments Details } \\
\text { Disorder Characteristic }\end{array}$} & \multirow{2}{*}{$\begin{array}{c}\text { vs Comparison } \\
\text { Primary Authors } \\
\text { (REVIEW Reference) }\end{array}$} & \multicolumn{4}{|c|}{ Quality of Evidence (GRADE*) } \\
\hline & & & Strong & Moderate & Low & Very Low \\
\hline \multicolumn{7}{|c|}{ EVIDENCE of NO BENEFIT ( $v s$ control) or No DIFFERENCE ( $v s$ another treatment) - Medical Injections and Oral Medication } \\
\hline 편 & $\begin{array}{l}\text { Meprobamate (psychotropic agent) } \\
\text { for acute neck disorder with } \\
\text { radiculopathy }\end{array}$ & $\begin{array}{l}\text { vs placebo } \\
\text { Payne } \text { et al. } 1964 \text { [72] } \\
\text { (PELOSO 2007 [7]) }\end{array}$ & & & IP pain & \\
\hline 팽: $\frac{0}{0}$ & $\begin{array}{l}\text { Fluoxetine (psychotropic agent) } \\
\text { for chronic WAD }\end{array}$ & $\begin{array}{l}v s \text { amitriptyline } \\
\text { Schreiber } \text { et al. 2001 [73] } \\
\text { (PELOSO 2007 [7]) }\end{array}$ & & & & IP pain \\
\hline 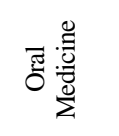 & $\begin{array}{l}\text { Chlormezanone (muscle relaxant) } \\
\text { for subacute non specific neck pain }\end{array}$ & $\begin{array}{l}\text { vs benorylate } \\
\text { Berry et al. } 1981 \text { [43] } \\
\text { (HURWITZ 2008 [44]) }\end{array}$ & & & & $\begin{array}{l}\text { ST pain, stiffness, sleep, } \\
\text { perceived effectiveness }\end{array}$ \\
\hline 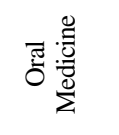 & $\begin{array}{l}\text { Chlormezanone (muscle relaxant) + } \\
\text { Benorylate (Analgesic) } \\
\text { for subacute non specific neck pain }\end{array}$ & $\begin{array}{l}\text { vs benorylate } \\
\text { Berry et al. } 1981 \text { [43] } \\
\text { (HURWITZ 2008 [44]) }\end{array}$ & & & & $\begin{array}{l}\text { ST pain, stiffness, sleep, } \\
\text { perceived effectiveness }\end{array}$ \\
\hline 胥: & $\begin{array}{l}\text { Chlormezanone (muscle relaxant) + } \\
\text { Benorylate (Analgesic) } \\
\text { for subacute non specific neck pain }\end{array}$ & $\begin{array}{l}\text { vs chlormezanone } \\
\text { Berry et al. } 1981 \text { [43] } \\
\text { (HURWITZ 2008 [44]) }\end{array}$ & & & & $\begin{array}{l}\text { ST pain, stiffness, sleep, } \\
\text { perceived effectiveness }\end{array}$ \\
\hline 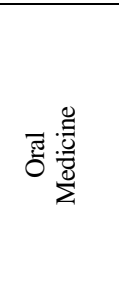 & $\begin{array}{l}\text { A) Celebrex, Vioxx (NSAIDs), } \\
\text { Paracetamol (analgesic) } \\
\text { B) Tenoxicam (NSAID) ranitidine } \\
\text { (histamine H2-receptor antagonist) } \\
\text { C) Diazepam (psychotropic) } \\
\text { for chronic specific neck pain }\end{array}$ & $\begin{array}{l}\text { vs acupuncture } \\
\text { A) Giles \& Muller } 2003 \\
\text { [74] } \\
\text { B) Giles \& Muller } 1999 \\
\text { [75] } \\
\text { C) Thomas et al. } 1991 \text { [64] } \\
\text { (FURLAN 2011 [68]; } \\
\text { FURLAN } 2012 \text { [76]) }\end{array}$ & & & & IP and ST pain \\
\hline 햄: & $\begin{array}{l}\text { A) Celebrex (NSAID), Vioxx } \\
\text { (NSAID), Paracetamol (analgesic) } \\
\text { B) Tenoxicam (NSAID) ranitidine } \\
\text { (Histamine H2-receptor antagonist) } \\
\text { for chronic specific neck pain }\end{array}$ & $\begin{array}{l}\text { vs manipulation } \\
\text { A) Giles \& Muller } 2003 \\
\text { [74] } \\
\text { B) Giles \& Muller } 1999 \\
\text { [75] } \\
\text { (FURLAN } 2011 \text { [68]; } \\
\text { FURLAN } 2012 \text { [76]) }\end{array}$ & & & & $\begin{array}{l}\text { IP and ST pain favour } \\
\text { manipulation } \\
\text { IP and ST disability (NDI } \\
\text { score) favour } \\
\text { manipulation }\end{array}$ \\
\hline 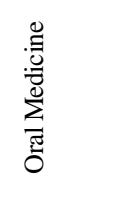 & $\begin{array}{l}\text { Treatments by GP (analgesics + anti- } \\
\text { inflammatory medications) + } \\
\text { Education } \\
\text { for subacute + chronic MND }\end{array}$ & $\begin{array}{l}\text { vs sham physical therapy } \\
\text { Koes et al. } 1992 \text { [77] } \\
\text { (PELOSO 2007 [7]; } \\
\text { HARALDSSON 2006 } \\
\text { [78]) }\end{array}$ & & & $\begin{array}{l}\text { ST, LT severity of } \\
\text { main complaint } \\
\text { ST, LT physical } \\
\text { function }\end{array}$ & \\
\hline 팽: & $\begin{array}{l}\text { Celebrex-celacoxin (NSAID), } \\
\text { Vioxx-rofecoxib (NSAID), } \\
\text { paracetamol (analgesic) } \\
\text { for chronic neck pain }\end{array}$ & $\begin{array}{l}\text { vs spinal manipulation } \\
\text { Muller } \text { et al. } 2005 \text { [79] } \\
\text { (GROSS 2010 [69]) }\end{array}$ & & & & $\begin{array}{l}\text { ST pain } \\
\text { ST function }\end{array}$ \\
\hline 팽: & $\begin{array}{l}\text { Piroxicam (Anti-inflammatory) } \\
\text { for chronic non specific neck pain } \\
\text { (Note: cervicobrachial pain - went to } \\
\text { original article) }\end{array}$ & $\begin{array}{l}\text { vs indomethacin } \\
\text { Yamamoto et al. } 1983 \text { [45] } \\
\text { (HURWITZ 2008 [44]) }\end{array}$ & & & $\begin{array}{l}\text { ST pain, physician } \\
\text { perceived } \\
\text { improvement }\end{array}$ & \\
\hline चूँ & $\begin{array}{l}\text { tenoxicam (NSAID) + ranitidine } \\
\text { (Histamine H2-receptor antagonist) } \\
\text { for chronic MND with degenerative } \\
\text { changes }\end{array}$ & $\begin{array}{l}\text { vs acupuncture or } \\
\text { manipulation } \\
\text { Giles \& Muller 1999 [74] } \\
\text { (PELOSO 2007 [7]; } \\
\text { GROSS 2010 [69]; } \\
\text { VERNON } 2007 \text { [80]) }\end{array}$ & & & & IP pain and function \\
\hline
\end{tabular}




\begin{tabular}{|c|c|c|c|c|c|c|}
\hline \multirow[t]{2}{*}{ Category } & \multirow{2}{*}{$\begin{array}{c}\text { Treatments Details } \\
\text { Disorder Characteristic }\end{array}$} & \multirow{2}{*}{$\begin{array}{c}\text { vs Comparison } \\
\text { Primary Authors } \\
\text { (REVIEW Reference) }\end{array}$} & \multicolumn{4}{|c|}{ Quality of Evidence (GRADE*) } \\
\hline & & & Strong & Moderate & Low & Very Lov \\
\hline 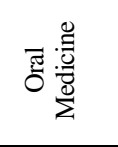 & $\begin{array}{l}\text { ibuprofen + manipulation } \\
\text { for chronic neck disorder with } \\
\text { headache and radiculopathy }\end{array}$ & $\begin{array}{l}\text { vs manipulation } \\
\text { Dostal et al. } 1978 \text { [81] } \\
\text { (PELOSO 2007 [7]) }\end{array}$ & & & IP pain & \\
\hline 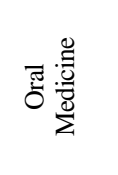 & $\begin{array}{l}\text { NSAIDs } \\
\text { for chronic specific neck pain }\end{array}$ & $\begin{array}{l}v s \text { acupuncture } \\
\text { Birch \& Jamison 1998 [82] } \\
\text { (FURLAN 2011 [76]; } \\
\text { VERNON 2009 [83]) }\end{array}$ & & & $\begin{array}{l}\text { IP pain favour } \\
\text { acupuncture }\end{array}$ & \\
\hline 정 & $\begin{array}{l}\text { NSAID } \\
\text { for chronic MND, neck disorder } \\
\text { with radicular signs }\end{array}$ & $\begin{array}{l}\text { vs continuous } \\
\text { traction and } \\
\text { exercise } \\
\text { Shakoor } \text { et al. } 2002 \text { [84] } \\
\text { (GRAHAM } 2006 \text { [85]) }\end{array}$ & & & IP pain & \\
\hline चूँ & $\begin{array}{l}\text { NSAIDs, placebo cervical traction } \\
\text { and postural advice } \\
\text { for chronic cervical spondylosis with } \\
\text { pain in neck and arms of root } \\
\text { distribution }\end{array}$ & $\begin{array}{l}v s \text { manual cervical traction, } \\
\text { exercise and postural } \\
\text { advice } \\
\text { Shakoor } \text { et al. } 2002 \text { [84] } \\
\text { (PEAKE 2005 [86]) }\end{array}$ & & & $\begin{array}{l}\text { IP pain } \\
\text { IP ROM } \\
\text { IP physician's } \\
\text { assessment of the } \\
\text { severity of the } \\
\text { conditions }\end{array}$ & \\
\hline 可 & $\begin{array}{l}\text { NSAIDs + Sham Acupuncture } \\
\text { for neck pain }\end{array}$ & $\begin{array}{l}v s \text { acupuncture } \\
\text { Birch \& Jamison } 1998 \text { [82] } \\
\text { (FU 2009 [87]) }\end{array}$ & & & $\begin{array}{l}\text { IP pain favor } \\
\text { acupuncture }\end{array}$ & \\
\hline 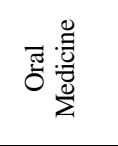 & $\begin{array}{l}\text { Glaphenine } \\
\text { for acute MND }\end{array}$ & $\begin{array}{l}v s \text { paracetamol } \\
\text { Choffray et al. } 1987 \text { [88] } \\
\text { (PELOSO 2007 [7]) }\end{array}$ & & & & $\begin{array}{l}\text { IP ROM } \\
\text { IP pain }\end{array}$ \\
\hline
\end{tabular}

Key: WAD - whiplash associated disorder; MND - mechanical neck disorder; NDI - neck disability index; GP - general practitioner; NSAID - nonsteroidal anti-inflammatory drug; $v s$ - versus; SF-36 - short form 36; GPE - global perceived effect; IP - immediate post treatment; ST - short term closest to 3 months, IT - intermediate term closest to 6 months, LT - long term closest to 1 year; ROM - range of motion; neg - negative findings or statistically not significant; pos- positive findings or statistically significant findings. AUTHORS OF SYSTEMATIC REVIEW shown in ALL CAPS. Authors of randomized trial shown without caps.

\section{FINAL EVIDENCE-BASED RECOMMENDATIONS (SEE TABLE 4)}

There is striking lack of trials and evidence for pharmacological therapies commonly used in neck pain. For subacute or chronic WAD, the evidence strongly recommends against the use of botulinum-A to reduce pain, improve disability or global perceived effect after short-term follow-up. For chronic facet joint pain and related disability, the evidence suggests against the use of medial branch block with steroids from short- to long-term follow-up data. For chronic neck pain, the evidence supports the use of only one muscle relaxant (psychotropic agent), eperison hydrochloride. There is limited efficacy with this agent however as it will help one in 37 people achieve immediate pain relief and evidence for longer-term benefits are not available.

\section{EVIDENCE OF BENEFIT}

This section provides data favouring the use of certain oral medication and medical injections by the GRADE of evidence.

Table 3. Therapies with Conflicting Evidence

\begin{tabular}{|c|c|}
\hline Treatments with Conflicting Evidence & Author (REVIEW) \\
\hline \hline $\begin{array}{c}\text { Nerve Block Injections } \\
\text { analgesic block of greater occipital nerve for neck disorder with } \\
\text { cervicogenic headache and radicular symptoms }\end{array}$ & $\begin{array}{c}\text { Terzi 2002 [91] (prilocaine } v s \text { saline) (positive findings) } \\
\text { Inan 2001 [92] (bupivacaine } v s \text { perineural injection) (negative findings) } \\
\text { (PELOSO 2007 [7]) }\end{array}$ \\
\hline
\end{tabular}


Table 4. Evidence-Based Recommendations

\begin{tabular}{|c|c|c|c|}
\hline $\begin{array}{l}\text { GRADE } \\
\text { Symbol }\end{array}$ & GRADE* ${ }^{*}$ and Recommendation & $\begin{array}{cc} & \text { Clinical Importance } \\
\text { - } & \text { Magnitude of Effect } \\
\text { - } & \text { Duration of Effect }\end{array}$ & Reported Adverse Effect or Side Effects \\
\hline$\infty$ & $\begin{array}{l}\text { Strong } \\
\text { Evidence of Benefit: } \\
\text { (Strongly recommend use) } \\
\text { No recommendation. } \\
\text { Evidence of NO Benefit: } \\
\text { (Strongly recommend against use) } \\
\text { 1) botulinum-A over saline placebo (5 trials, } \\
\quad \text { 258 participants) for chronic non-specific } \\
\text { neck pain for short-term pain. }\end{array}$ & $\begin{array}{l}\text { NA } \\
\text { Meta-analysis: } \\
\text { ST Pain: SMDp }-0.07 \\
\text { (95\% CI }-0.36 \text { to } 0.21 \text { ) }\end{array}$ & $\begin{array}{l}\text { NA } \\
\text { Minor, transient and reversible: excessive } \\
\text { weakness of injected muscle, arm heaviness and } \\
\text { numbness, transient pain or soreness at injection } \\
\text { site, flu like symptoms, shift of pain }\end{array}$ \\
\hline$\bullet$ & $\begin{array}{l}\text { Moderate } \\
\text { Evidence of Benefit: } \\
\text { (Suggested use) } \\
\text { 1) oral psychotropic agent, eperison } \\
\text { hydrochloride, compared to placebo (1 } \\
\text { trial, 157 participants) for chronic MND } \\
\text { to improve pain and range of motion at } \\
\text { immediate post treatment }\end{array}$ & $\begin{array}{l}\text { IP Pain: RR } 0.68 \\
\text { (95\%CI } 0.52 \text { to } 0.90) \\
\text { NNT } 37\end{array}$ & NR \\
\hline 0000 & $\begin{array}{l}\text { Moderate } \\
\text { Evidence of NO Benefit: } \\
\text { (Suggested not to use) } \\
\text { 1) medial branch block with steroid } v s \\
\text { control (1 trials, } 120 \text { participants) } \\
\text { medial branch block with steriod } \\
\text { a) bupivacaine + sarapin } \\
\text { b) bupivacaine+betamethasone } \\
\text { c) bupivacaine+betamethasone + } \\
\text { over bupivacaine for chronic cervical facet } \\
\text { joint pain at short and long term follow-up }\end{array}$ & $\begin{array}{l}\text { LT Pain (NRS): WMD }-0.30 \text { ( } 95 \% \text { CI } \\
-0.68 \text { to } 0.08) \\
\text { LT Disability } \dagger \text { (NDI): WMD } 0.00 \\
\text { ( } 95 \% \text { CI }-1.72 \text { to } 1.72 \text { ) }\end{array}$ & $\begin{array}{l}\text { Transient facial flushing and temporary } \\
\text { exacerbation of usual pain }\end{array}$ \\
\hline ০০ & $\begin{array}{l}\text { 2) botulinum-A over placebo ( } 4 \text { trials, } 183 \\
\text { participants) for subacute or chronic WAD to } \\
\text { reduce pain, disability or global perceived } \\
\text { effect at short-term follow-up. }\end{array}$ & $\begin{array}{l}\text { ST Pain: SMDp }-0.21 \\
\text { (95\% CI }-0.57 \text { to } 0.15 \text { ) } \\
\text { ST Disability: SMDp } 0.15 \\
\text { (95\% CI }-0.37 \text { to } 0.68 \text { ) } \\
\text { ST GPE: SMDp } 0.15 \\
\text { (95\% CI }-0.37 \text { to } 0.68 \text { ) }\end{array}$ & $\begin{array}{l}\text { Minor, transient and reversible: } \\
\text { excessive weakness of injected muscle, arm } \\
\text { heaviness and numbness, transient pain or } \\
\text { soreness at injection site, flu like symptoms, } \\
\text { shift of pain }\end{array}$ \\
\hline
\end{tabular}

GRADE*: study design, within study risk of bias, consistency of results, directness (generalizability), precision (sufficient data), reporting bias (publication, language, funding, other); open symbol= no benefit; closed symbol = beneficial; duration of effect noted by number of symbols: one $=$ IP, two $=$ ST, three $=$ IT, $4=\mathrm{LT}$; diamond $(\bullet)=$ high GRADE; $\operatorname{dot}(\bullet)=$ moderate GRADE.

Clinically Important is determined by considering the following factors: minimal detectable change, minimal clinically important difference ( $\geq 15 \%)$, large magnitude of effect (weighted mean difference, number needed to treat, absolute benefit, treatment advantage), high dose response gradient, duration of the effect (IP - immediate post treatment, ST short term for about 3 months, IT - intermediate term for about 6 months, LT - long term for about 1 year).

Key: WAD - whiplash associated disorder; MND - mechanical neck disorder; SMDp - Standard Mean Difference pooled; WMD - weighted mean difference; RR - relative risk; NNT - number-needed-to-treat; 95\%CI - 95\% confidence interval, $\dagger$ no significant difference between groups for this outcome, GPE - global perceived effect; NR - not reported; NA - not applicable.

\section{Strong Evidence of Benefit}

Based on our assessment, we found no trials meeting criteria for this strength of evidence.

\section{Moderate Evidence of Benefit}

\section{Medicinal Injection}

There were no medicinal injections that met the criteria for moderate quality evidence of benefit.

\section{Oral Medication}

\section{Psychotropic}

We found one trial with 215 participants [42] favouring a small benefit [NNT 37, RR 0.68 (95\% CI 0.52 to 0.90$)$ ] with eperison hydrochloride, a muscle relaxant/psychotropic agent, relative to placebo in patients with chronic mechanical neck disorder at immediate post treatment. There were no reported benefits on pain and range of motion. 
Table 5. AMSTAR Rating of Medicine Reviews for Neck Pain

\begin{tabular}{|c|c|c|c|c|c|c|c|c|c|c|c|}
\hline Author & 1 & 2 & 3 & 4 & & 6 & 7 & 8 & 9 & 10 & 11 \\
\hline Abdi et al. 2005 [32] & $\mathrm{Y}$ & $\mathrm{N}$ & $\mathrm{Y}$ & $\mathrm{Y}$ & $\mathrm{Y}$ & $\mathrm{Y}$ & $\mathrm{Y}$ & $\mathrm{Y}$ & NA & $\mathrm{N}$ & $\mathrm{Y}$ \\
\hline Benyamin et al. 2009 [30] & $\mathrm{Y}$ & $\mathrm{N}$ & $\mathrm{Y}$ & $\mathrm{N}$ & $\mathrm{N}$ & $\mathrm{Y}$ & $\mathrm{Y}$ & $\mathrm{Y}$ & NA & $\mathrm{N}$ & $\mathrm{N}$ \\
\hline Boswell et al. 2005 [35] & $\mathrm{Y}$ & $\mathrm{N}$ & $\mathrm{Y}$ & $\mathrm{N}$ & $\mathrm{N}$ & $\mathrm{Y}$ & Y & $\mathrm{Y}$ & NA & $\mathrm{N}$ & $\mathrm{Y}$ \\
\hline Conlin et al. 2005 [18] & $\mathrm{Y}$ & $\mathrm{N}$ & $\mathrm{Y}$ & $\mathrm{N}$ & $\mathrm{N}$ & $\mathrm{Y}$ & $\mathrm{Y}$ & $\mathrm{Y}$ & $\mathrm{Y}$ & $\mathrm{N}$ & $\mathrm{N}$ \\
\hline Falco etal 2009 [36] & $\mathrm{Y}$ & Y & $\mathrm{Y}$ & $\mathrm{N}$ & $\mathrm{N}$ & $\mathrm{Y}$ & $\mathrm{Y}$ & $\mathrm{Y}$ & NA & $\mathrm{N}$ & $\mathrm{N}$ \\
\hline Fu et al. 2009 [87] & $\mathrm{Y}$ & $\mathrm{Y}$ & $\mathrm{Y}$ & $\mathrm{N}$ & $\mathrm{Y}$ & $\mathrm{Y}$ & $\mathrm{Y}$ & $\mathrm{N}$ & $\mathrm{Y}$ & $\mathrm{N}$ & $\mathrm{N}$ \\
\hline Furlan et al. 2011 [68] & Y & Y & Y & Y & $\mathrm{Y}$ & Y & $\mathrm{Y}$ & Y & $\mathrm{Y}$ & $\mathrm{N}$ & $\mathrm{N}$ \\
\hline Gross et al. 2010 [69] & $\mathrm{Y}$ & Y & $\mathrm{Y}$ & $\mathrm{Y}$ & $\mathrm{Y}$ & $\mathrm{Y}$ & $\mathrm{Y}$ & $\mathrm{Y}$ & $\mathrm{Y}$ & $\mathrm{N}$ & $\mathrm{N}$ \\
\hline Haraldsson et al. 2006 [78] & $\mathrm{Y}$ & $\mathrm{Y}$ & $\mathrm{Y}$ & $\mathrm{Y}$ & $\mathrm{Y}$ & $\mathrm{Y}$ & $\mathrm{Y}$ & $\mathrm{Y}$ & $\mathrm{Y}$ & $\mathrm{N}$ & $\mathrm{N}$ \\
\hline Hurwitz et al. 2008 [44] & Y & $\mathrm{N}$ & $\mathrm{N}$ & Y & $\mathrm{N}$ & Y & $\mathrm{Y}$ & Y & NA & $\mathrm{N}$ & $\mathrm{N}$ \\
\hline Langevin et al. 2011 [26] & $\mathrm{Y}$ & Y & $\mathrm{Y}$ & $\mathrm{Y}$ & $\mathrm{Y}$ & $\mathrm{Y}$ & $\mathrm{Y}$ & $\mathrm{Y}$ & $\mathrm{Y}$ & $\mathrm{N}$ & $\mathrm{N}$ \\
\hline Langevin et al. 2011 [90] & $\mathrm{Y}$ & Y & $\mathrm{Y}$ & $\mathrm{Y}$ & $\mathrm{Y}$ & $\mathrm{Y}$ & $\mathrm{Y}$ & $\mathrm{Y}$ & $\mathrm{Y}$ & $\mathrm{N}$ & $\mathrm{N}$ \\
\hline Leaver et al. 2010 [48] & $\mathrm{Y}$ & $\mathrm{Y}$ & $\mathrm{Y}$ & $\mathrm{N}$ & $\mathrm{N}$ & $\mathrm{Y}$ & $\mathrm{Y}$ & $\mathrm{Y}$ & $\mathrm{Y}$ & $\mathrm{N}$ & $\mathrm{N}$ \\
\hline Leite et al. 2009 [71] & $\mathrm{Y}$ & Y & Y & $\mathrm{Y}$ & $\mathrm{N}$ & Y & $\mathrm{Y}$ & Y & NA & $\mathrm{N}$ & $\mathrm{N}$ \\
\hline Peake \& Harte 2005 [86] & $\mathrm{Y}$ & $\mathrm{N}$ & Y & $\mathrm{N}$ & $\mathrm{N}$ & Y & $\mathrm{Y}$ & Y & NA & $\mathrm{N}$ & $\mathrm{N}$ \\
\hline Vernon \& Schneider 2009 [83] & $\mathrm{Y}$ & $\mathrm{N}$ & $\mathrm{Y}$ & $\mathrm{N}$ & $\mathrm{N}$ & $\mathrm{N}$ & $\mathrm{Y}$ & $\mathrm{Y}$ & NA & $\mathrm{N}$ & $\mathrm{N}$ \\
\hline
\end{tabular}

Key: Y Yes; N No; NA not applicable; CA can`t assess; AMSTAR Questions:

1. Was an 'a priori' design provided? The research question and inclusion criteria should be established before the conduct of the review.

2. Was there duplicate study selection and data extraction? There should be at least two independent data extractors and a consensus procedure for disagreements should be in place.

3. Was a comprehensive literature search performed? At least two electronic sources should be searched. The report must include years and databases used (e.g. Central, EMBASE, and MEDLINE). Key words and/or MESH terms must be stated and where feasible.

4. Was the status of publication (i.e. grey literature) used as an inclusion criterion? The authors should state that they searched for reports regardless of their publication type. The authors should state whether or not they excluded any reports.

5. Was a list of studies (included and excluded) provided? A list of included and excluded studies should be provided.

6. Were the characteristics of the included studies provided? In an aggregated form such as a table, data from the original studies should be provided on the participants, interventions and outcomes. The ranges of characteristics in all the studies analyzed eg age, race, sex relevant socioeconomic data, disease status, duration, severity, or other diseases should be reported.

7. Was the scientific quality of the included studies assessed and documented? 'A priori' methods of assessment should be provided (e.g., for effectiveness studies if the author(s) chose to include only randomized, double-blind, placebo controlled studies or allocation concealment as includion criteria); for other types of studies alternative items will be relevant.

8. Was the scientific quality of the included studies used appropriately in formulating conclusions? The results of the methodological rigor and scientific quality should be considered in the analysis and the conclusions of the review, and explicitly stated in formulating recommendations.

9. Were the methods used to combine the findings of studies appropriate? For the pooled results, a test should be done to ensure the studies were combinable, to assess their homogeneity (i.e. Chi-squared test for homogeneity, 2). If heterogeneity exists a random effects model should be used and/or the clinical appropriateness of combining should be taken into consideration (i.e. is it sensible to combine?)

10. Was the likelihood of publication bias assessed? An assessment of publication bias should include a combination of graphical aids (e.g., funnel plot, other available tests) and/or statistical tests (e.g., Egger regression test)

11. Was the conflict of interest stated? Potential sources of support should be clearly acknowledged in both the systematic review and the included studies. 
Table 6. Harms Summary of Findings with AMSTAR Score

\begin{tabular}{|c|c|c|c|c|c|}
\hline Category & $\begin{array}{c}\text { Treatment Details } \\
\text { Disorder Characteristic }\end{array}$ & Review Reference & $\begin{array}{l}\text { AMSTAR Score } \\
\text { and Quality }\end{array}$ & Adverse Events & Frequency \\
\hline 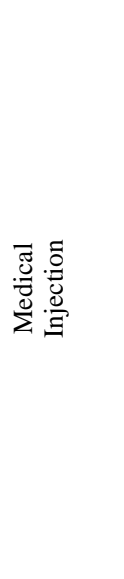 & $\begin{array}{l}\text { Epidural steroid- } \\
\text { Cervical axial or } \\
\text { radicular pain } \\
\text { Transforaminal }\end{array}$ & $\begin{array}{l}\text { Malhotra } 2009 \text { [93] } \\
\text { Guzman } 2008 \text { [94] } \\
\text { Abdi } 2005 \text { [95] }\end{array}$ & $\begin{array}{l}2 \text { - low } \\
4 \text { - low } \\
7 \text { - high }\end{array}$ & $\begin{array}{l}\text { Headache, } \\
\text { Transient neurological deficits (pain, } \\
\text { weakness) } \\
\text { Hypersensitivity reaction, } \\
\text { Vasovagal response, } \\
\text { Nausea } \\
\text { Transient global amnesia } \\
\text { Allergic responses } \\
\text { Seizure } \\
\text { Spinal cord, brainstem or brain edema } \\
\text { Cortical blindness, } \\
\text { Epidural or paraspinal hematoma } \\
\text { Peripheral neuropraxia } \\
\text { Dural puncture } \\
\text { Cervical spinal cord or vertebrobasilar } \\
\text { infract } \\
\text { Transient ischemia attack } \\
\text { Death }\end{array}$ & $\begin{array}{l}0 \text { to } 22.7 \% \text { for minor } \\
\text { transient adverse } \\
\text { events. No values } \\
\text { reported for all } \\
\text { others. }\end{array}$ \\
\hline 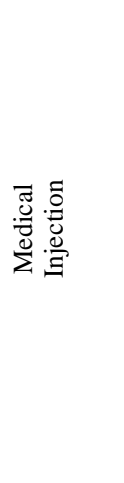 & $\begin{array}{l}\text { Epidural steroid - } \\
\text { Cervical axial or } \\
\text { radicular pain } \\
\text { Interlaminar }\end{array}$ & $\begin{array}{l}\text { Abbasi } 2007 \text { [96] } \\
\text { Guzman } 2008 \text { [94] } \\
\text { Abdi } 2005 \text { [95] }\end{array}$ & $\begin{array}{l}2 \text { - low } \\
4 \text { - low } \\
7 \text { - high }\end{array}$ & $\begin{array}{l}\text { Retinal hemorrhage } \\
\text { Allergic reaction } \\
\text { Epidural hematoma } \\
\text { Subdural complications } \\
\text { Dural puncture } \\
\text { Headache neuropathic symptoms } \\
\text { Intracranial hypotension and epidural } \\
\text { granuloma } \\
\text { Permanent spinal cord injury } \\
\text { Intravascular uptake of injectate } \\
\text { Pneumocephalus } \\
\text { Venous air embolism } \\
\text { Cervical epidural abscess } \\
\text { Cushing's syndrome } \\
\text { Death }\end{array}$ & $\begin{array}{l}0 \text { to } 17 \% \text { for minor } \\
\text { transient adverse } \\
\text { events. No values } \\
\text { reported for all } \\
\text { others. }\end{array}$ \\
\hline 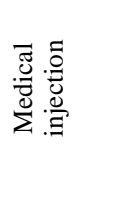 & $\begin{array}{l}\text { Stellate ganglion block } \\
\text { Sympathetically } \\
\text { maintained pain }\end{array}$ & Higa 2006 [97] & 3 - low & $\begin{array}{l}\text { Retropharyngeal hematoma causing } \\
\text { airway blockage can lead to death } \\
\text { - precipitated by head, neck or chest } \\
\text { pain } \\
\text { dyspnea, neck swelling, abnormal } \\
\text { sensations in the upper airway }\end{array}$ & $\begin{array}{l}\text { Overall rate } 14 / 27 \\
\text { patients }(52 \%) \\
21(78 \%) \text { requiring } \\
\text { airway management } \\
1(3.7 \%) \text { death }\end{array}$ \\
\hline 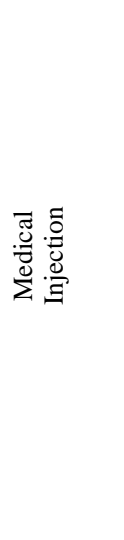 & $\begin{array}{l}\text { Intraarticular facet joint, } \\
\text { medial branch block, } \\
\text { medial branch } \\
\text { radiofrequency } \\
\text { neurotomy } \\
\text { Chronic spinal pain 3-6 } \\
\text { months in duration }\end{array}$ & $\begin{array}{l}\text { Boswell } 2005 \text { [98] } \\
\text { Guzman } 2008 \text { [94] }\end{array}$ & $\begin{array}{l}5 \text { - low } \\
4 \text { - low }\end{array}$ & $\begin{array}{l}\text { Dural puncture, } \\
\text { spinal cord trauma, infection, } \\
\text { spinal anesthesia, chemical meningitis, } \\
\text { neural trauma, } \\
\text { pneumothorax, } \\
\text { radiation exposure, } \\
\text { facet capsule rupture, } \\
\text { hematoma formation, and } \\
\text { steroid side effects } \\
\text { Radiofrequency neurotomy - } \\
\text { Cutaneous dysthesisas } \\
\text { Neuritis/neurogenic inflammation, } \\
\text { Anesthesia dolorosa } \\
\text { Cutaneous hyperesthesia, } \\
\text { pneumothorax } \\
\text { Deafferent pain }\end{array}$ & $\begin{array}{l}\text { Overall rate not } \\
\text { reported }\end{array}$ \\
\hline
\end{tabular}

\section{Low or Very Low Evidence of Benefit}

\section{Medicinal Injections}

Limited data from low to very low GRADE evidence suggests there may be benefit in the use of the following five medicinal injections: $\underline{\text { Paralytic }}$

1. Botulinum toxin-A plus exercise/medication combination for subacute/chronic WAD [23] or nonspecific neck pain for intermediate term pain (metaanalysis $[24,25])$. 


\section{$\underline{\text { Intramuscular (IM) Analgesic }}$}

2. Intramuscular lidocaine injection with or without stretching (versus placebo [19]; versus dry needling [21]) for chronic non-specific mechanical neck pain for short-term pain.

Interlaminar Cervical Epidural Steroid

3. Epidural steroids (plus lidocaine [27, 29]; plus morphine [28]) versus various control injections for chronic neck mechanical neck pain with radiculopathy or radiation into the arm for intermediate-term [29] to long-term pain, function and return to work.

\section{Intravenous Corticosteroid}

4. Methylprednisolone versus placebo for intermediateterm sick leave and disabling symptoms [17], in an acute emergency room WAD population.

Subcutaneous Saline

5. Subcutaneous sterile water injection may be beneficial in reducing pain for chronic neck pain after whiplash [37] based on very low quality evidence.

\section{Oral Medications}

Limited evidence from low to very low GRADE evidence suggests there may be benefit with the use of the following 10 oral medications:

\section{Psychotropic/Muscle Relaxant}

1. Cyclobenzaprine plus lysinine cloniximate versus lysinine cloniximate for subacute nonspecific mechanical neck disorder for immediate post treatment and short-term pain [40].

2. Tetrazepam plus paracetamol versus paracetamol alone for acute non-specific mechanical neck disorder for immediate post treatment pain, range of motion and global perceived effect [41].

3. Chlormezanone versus placebo for subacute nonspecific neck pain for immediate post treatment sleep [43].

\section{Anti-Inflammatory}

4. Piroxicam versus placebo for chronic non-specific pain for short-term pain, for physician perceived improvement [45].

5. Indomethacin versus placebo for non-specific neck pain for short-term pain, for physician perceived improvement [45].

6. Tolmetin versus naproxen for non-specific mechanical neck disorder and osteoarthritis for immediate post treatment pain and range of motion [46].

\section{$\underline{\text { Analgesic }}$}

7. Benorylate versus placebo for subacute to chronic nonspecific neck pain for immediate post treatment pain, stiffness, sleep and ability to work [43].

8. Benorylate plus chlormezanone versus placebo for subacute non-specific neck pain for immediate post treatment pain [43].
9. Norgesic (paracetamol plus orphenadrine) versus placebo for non-specific neck pain for short-term pain [47].

10. Oxycodone controlled release versus placebo for nonspecific neck pain for short-term pain, frequency of patients' pain episodes, quality of life and sleep [49].

\section{EVIDENCE OF NO BENEFIT}

\section{Strong Evidence of No Benefit}

\section{Medical Injection}

Paralytic

For botulinum toxin-A (1 review [26] conducted a metaanalysis of 4 trials, including 183 participants [24, 50, 51, 52]), finding no benefit over placebo for chronic non-specific neck pain at short term follow-up.

\section{Moderate Evidence}

\section{Medical Injection}

$\underline{\text { Paralytic }}$

For botulinum toxin-A, one review [26] conducted a metaanalysis of 4 trials (122 participants [23, 56, 57, 58]) and found no benefit over placebo for patients with subacute/chronic WAD in pain relief, disability or global perceived effect at short-term follow-up.

\section{Corticosteroids}

For a medial branch block with steroid added to bupivacaine versus bupivacaine alone (1 trial [33, 34]; 60 participants), we found evidence of small benefits in pain, but not for function for chronic cervical facet joint pain in the short-, intermediate- and long-term [long-term pain: WMD -0.30 (95\% CI -0.68 to 0.08)]. This result was not statistically significant and not likely to be clinically important; this trial was performed in a population with severe pain.

\section{Low or Very Low Evidence of No Benefit \\ Medicinal Injection}

We found limited information based on low to very low GRADE evidence suggesting there is no evidence of benefit for the following 4 medical injections:

\section{Corticosteroids}

Intra-articular steroid injection versus anaesthetics for chronic WAD for intermediate-term pain [60].

Paralytic

1. Botulinum toxin-A versus placebo for chronic cervicogenic headache pain (2 trials 58 participants [53, 54] and disability (1 trial [53]) at short-term and 1 trial [53] at intermediate-term follow-up. Additionally botulinum toxin-A versus placebo (saline) (1 trial [55], 45 participants) was not beneficial in improving disability or global perceived effect for chronic myofascial neck and shoulder pain at intermediate-term follow-up.

\section{Subcutaneous Insufflation}

2. Subcutaneous insufflation of $\mathrm{CO}_{2}$ plus physiotherapy versus physiotherapy alone for immediate post treatment pain [63]. 


\section{$\underline{\text { Intramuscular Injection Vitamin }}$}

3. Intramuscular injection of neurotropic multivitamin plus analgesic versus analgesic alone for chronic neck disorder with radicular symptoms for immediate post treatment pain and global perceived effect [59].

\section{Oral Medication}

The authors noted limited information (low to very low GRADE) suggesting no evidence of benefit for the following 17 oral medications:

\section{Psychotropic - Benzodiazepines and Muscle Relaxants}

1. Diazepam for acute mechanical neck disorder with spasm [66], subacute mechanical neck disorder with possible radicular symptoms [65], non-specific neck pain and chronic cervical degeneration [64] for immediate post treatment pain [64] and short-term pain, tenderness relief [66] and global evaluation of muscle spasm [65].

2. Diazepam versus manipulation for chronic nonspecific neck pain for short-term pain and functional improvement [67].

3. Cyclobenzaprine (versus placebo [65]; versus lidocaine infiltration [70] for subacute MND or trapezius myofascial pain for short-term global evaluation of muscle spasm and global pain and pain at digital compression.

4. Phenobarbitol for acute MND for short-term pain and tenderness [66].

5. Meprobamate versus placebo for acute neck disorder with radiculopathy for immediate post treatment pain [72].

6. Fluoxetine versus amitriptyline for chronic WAD for immediate post treatment pain [73].

7. Chlormezanone (versus benorylate); chlormezanone plus benorylate (versus benorylate); chlormezanone plus benorylate (versus chlormezanone) for subacute non-specific neck pain for short-term pain, stiffness, sleep and perceived effectiveness [43].

$\underline{\text { Anti-Inflammatory Plus Analgesic }}$

1. Celecoxib, rofecoxib, paracetamol [74], tenoxicam [75] and diazepam [64] versus acupuncture for chronic specific neck pain for immediate post treatment and short-term pain.

2. Celecoxib, rofecoxib, paracetamol [43], tenoxicam [75] versus manipulation for chronic specific neck pain for immediate post treatment and short-term pain and disability. (Note: At immediate post treatment and at short-term follow-up, manipulation was favored for pain and disability outcomes).

3. Treatments by a general practitioner (analgesics plus anti-inflammatory medications) plus education versus sham physical therapy for subacute and chronic MND for short-term to long-term severity of main complaint and physical function [78].
4. Celecoxib, rofecoxib, paracetamol versus spinal manipulation for chronic neck pain for short-term pain and function [80].

5. Piroxicam versus indomethacin for chronic nonspecific neck pain for short-term pain and physician perceived improvement [45].

6. Ibuprofen plus manipulation versus manipulation for chronic neck disorder with headache and radiculopathy for immediate post treatment pain [82].

7. NSAIDs for chronic specific neck pain (versus acupuncture [83]), chronic MND and neck disorder with radicular signs (versus continuous traction and exercise [86]) for pain relief immediately post treatment. (Note: At immediate post treatment, acupuncture was favored for the pain outcome).

8. NSAIDs (plus placebo cervical traction and postural advice [86]; plus sham acupuncture [83]) versus manual cervical traction, exercise and postural advice [86]; acupuncture [83] for chronic cervical spondylosis with pain in neck and arms of root distribution and neck pain for immediate post treatment pain, range of motion and physician's assessment of the severity of the conditions. (Note: Pain reduction favored acupuncture at immediate post treatment).

Analgesic

Glaphenine versus paracetamol for acute MND for immediate post treatment pain and range of motion [88].

$\underline{\text { Other }}$

Melatonin versus placebo for chronic MND or WAD for immediate post treatment pain, sleep and health status [89].

\section{Adverse Events \\ Medicinal Injections}

This section first discusses medicinal injections and then medicines. In the reviews themselves that informed on efficacy, we found minor, transient and reversible side effects following injections, including increased pain reporting for several hours to several days post injection. However a valid estimate of clinically important, uncommon, and rare adverse events cannot be made from these trials due to limited reporting in the original trials and in the reviews. With botulinum toxin-A injection, excessive weakness of the injected muscle, arm heaviness and numbness, transient pain or soreness at injection site, flu like symptoms and shift of pain occurred [7]. For bupivacaine nerve block injection, transient facial flushing and temporary exacerbation of usual pain were reported [7] as they were also reported for intra-articular use of betamethasone [60]. Injection pain, allergic reaction and headache were associated with the administration of intramuscular multivitamin plus analgesic [59]. Worsened pain was associated with both epidural steroid and lidocaine injections [7, 27]. Malaise, headache, nausea and vomiting were associated with subcutaneous $\mathrm{CO}_{2}$ used with physical therapy [63]. 
We also performed searches specific to harms to augment the information found in the reviews. As part of these searches we found evidence of both minor transient and major catastrophic adverse events for injections [93-98]. Table 6 lists the adverse events associated with each procedure. For both transforaminal and interlaminar epidural steroid injections there is a broad list of adverse events, with interlaminar injections appearing to have more serious outcomes, possibly since the technique approaches the spinal cord more directly [99]. The review on stellate ganglion blocks [94] only considered the development of retropharyngeal hematoma and not other adverse events associated with this procedure. Reviews of intraarticular facet joint and medial branch injections adverse events reported mainly major adverse outcomes, with Higa et al. (2006) reporting an adverse outcome of death (1 death or $3.7 \%$ of population) [97]. Injections appear to lead to minor adverse events occur in approximately 1 in 5 patients. However limited reporting overall hinders the ability to provide a more precise estimate of injections' safety.

\section{Oral Medications}

Based on the treatment reviews, a valid estimate of clinically important, uncommon, and rare adverse events is not possible, due to limited reporting on adverse events. Minor side effects were reported with oral medications in some trials. Sleepiness was associated with taking diazepam [66]; drowsiness, mouth dryness and xerostomia were associated with taking cyclobenzaprine $[65,70]$; sleepiness, gastrointestinal upset and skin irritation were associated with taking cyclobenzaprine and lysinine cloniximate [40]; dizziness, fatigue and dry mouth were associated with taking tetrazepam and paracetamol [41]; dizziness and drowsiness were reported for taking phenobarbitol [66]; drowsiness, nausea and indigestion were reported for taking meprobamate [72]; fluoxetine was reported to have anticholinergic effects such as dryness and dizziness [73]; dyseptic difficulties, elimination difficulties and drowsiness were associated with taking glaphenine [89]; drowsiness, cephalalgia, dyspeptic difficulties, ulcer and vertigo were reported for taking paracetamol [89]; headaches were associated with taking melatonin [89].

Importantly, no systematic reviews presenting the harms of oral medications in the neck pain population were identified, and therefore is speculation whether an events and event rates seen in other populations might also apply to the neck pain population.

\section{DISCUSSION}

There continues to be a lack of high-quality evidence to inform recommendations on the use of medicinal injections and medicines for neck pain. Only one trial meeting the moderate quality evidence threshold and reporting evidence of benefit was uncovered. The trial by Bose et al. supported the use of an oral psychotropic agent, eperison hydrochloride, for subjects with chronic mechanical neck disorder for immediate post treatment pain and range of motion but longer term follow-up was not reported and the treatment effects were small (1 expected to benefit for 37 treated).
There was more data on evidence of no benefit. In the review by Langevin et al. [26], a meta-analysis of four trials provided a strong quality of evidence demonstrating no benefit for botulinum toxin-A over placebo for subjects with chronic non-specific neck pain for short-term pain. Additionally in the same review by Langevin et al, another meta-analysis of four trials with moderate quality evidence demonstrated no benefit for botulinum toxin-A over placebo for subacute or chronic WAD patients for short-term pain, disability or global perceived effect. Two reports of one trial by Manchikanti et al. [33, 34] did not support the use of nerve block injections with bupivacaine and varying combinations of steroid and sarapin for subjects with cervical facet joint pain for short- and long-term pain.

Although oral medication such as analgesics, antiinflammatories, antidepressants, opioids, psychotropic, and muscle relaxants are commonly used in clinical practice, there continues to be low to very low quality evidence available for their benefits and risks in neck pain. Furthermore the existing evidence is conflicting, which limits the ability to make clear recommendations. Data on disability, function, and quality of life are rarely reported. Most pharmacological therapies would be expected to produce side effects and their balance of risks and benefits are likely to vary by the condition being treated. We speculate that physicians assume that injections and medications demonstrating efficacy for other musculoskeletal conditions such as in low back pain [100] inform their use in neck pain and that clinical trials are not being conducted or not considered necessary in the neck pain population. However we are not aware of data that suggests treatment benefits seen in the back pain population can be extended to the neck pain populatoin. Given that neck pain is common, potentially disabling and costly to society, and that benefit-risk may well vary by condition, high quality studies are still needed to understand the benefits and risks in the neck pain population. Our qualitative research suggests that the side effects associated with medication use can be very concerning for patients and patients may discontinue medications related to these concerns [101]. Specifically patient's worried about how medications would interfere with their ability to participate in normal life roles [101]. Further, patients in our qualitative study indicated that they prefer that physicians present all treatment options and not confine their recommendations to prescriptions of medications alone [101].

For medicinal injections, the therapies with the most supporting evidence continue to be IV methylprednisolone for acute whiplash, IM-lidocaine for chronic MND, and epidural methylprednisonlone with lidocaine injection for radiculopathy. It remains unclear if all corticosteroids or local anesthetics are equally effective or if there is a dose response for these therapies. Replication in larger, high quality trials is needed for these injections. If subsequent trials were positive, efforts to promote widespread adoption would be indicated. Anti-inflammatory drugs warrant further study particularly since several of them such as ibuprofen and naproxen may be available over the counter without a prescription; we note that they are also frequently used as cointervention with other physiotherapy management approaches. Oral psychotropic agents classified as muscle relaxants, such as cyclobenzaprine, diazepam, and 
tetrazepam continue to require further study to clarify their benefits and harms. There were no studies of tricyclic antidepressants and one very low quality trial on opiate analgesics in chronic neck pain. In this regard, little has changed since the 1996 [102] and 2007 [20] systematic reviews.

Another challenge in making more definitive recommendations was the lack of high-quality clinical trials that addressed meaningful outcomes in a standardized way. Many studies focus on pain alone with function being reported to a lesser extent. Even when function is reported, different outcomes are used. Consistent use of pain and disability outcomes would facilitate cross study comparisons and inform future metaanalyses. A number of reporting and design issues in neck pain clinical trials have been detailed in Goldsmith et al. [103]. There are design features that would improve the quality of future clinical trials in neck pain. In particular, future research should ensure adherence to CONSORT guidelines, look beyond the basic two group design (active $v s$ placebo or active $v s$ active) that is commonly used and the should consistently report standardized impairment and disability outcomes [104]. A core set of patient reported outcomes and key participation indicators (such as return to work) are needed. Further an accurate prospective collection of adverse events is also fundamental. Finally, studies that compare medicines or injections to other commonly used therapies, such as physical therapies and manual therapies are needed to understand whether some therapies should preferentially be recommended.

The ability to generalize our findings across the entirety of the neck pain population is limited from at least four perspectives. First, there are many disorders that can have neck pain as an associated feature, such as migraine, tension type headache and trigeminal neuralgia. A careful differential diagnosis and its reporting are important when describing neck pain patients included in any trial. This report specifically applies to a narrow type of mechanical neck pain with or without cervicogenic headache or radiculopathy. Second, when considering botulinum toxin injections, identification of the dystonic muscle may need EMG guidance to ensure the right region or muscles are injected; this was not the case in the trials included in this review. Third, once neck pain has been identified, the mechanisms underpinning the pain experience need to be identified where possible. Mechanisms underlying peripheral or central neuropathic pain will be treated differently than nociceptive mechanisms such as chemical [inflammatory from tissue (bradykinin, prostaglandin, serotonin), nerve (NGK, neurokinins, noradrenaline), immune cells (macrophages, cytokines)], ischaemic (SP, CGRP) and mechanical. Understanding the pain experience could help to optimize different medical strategies. Finally, understading why some patients respond and other do not would also allow the most appropriaat therapy to be directed to the right patients. Clinical decision rules may then help guide practice.

Our approach to summarizing the literature has several strengths. We used a comprehensive, librarian-assisted search of multiple databases. We used two independent reviewers to determine article relevance and assess review quality using the AMSTAR tool. We used at least two people to verify data extraction. We used a group and an external panel consensus approach to validate the GRADE of evidence and recommendations. We avoided the professional bias inherent in having a single professional group evaluate its own literature. The largest limitation is that new trials will have been published following the publication of many of these reviews. Given the state of the literature, one large trial with a low risk of bias could change the direction of benefit (positively or negatively) as well as the magnitude of that benefit. We did consider the potential for selection bias and we examined the grey literature of reviews as part of our search strategy.

The vast majority of trials did not lead to firm recommendation, since few injections and medicines results have been replicated by large, high quality trials. Although we did find some meta-analyses (the highest level of evidence), the quality of studies for neck pain continues to be limited. Adverse effects and associated costs of treatment are widely under-reported and when they are reported are they often in narrative form rather than quantitatively expressed. There is evidence of major catastrophic adverse events with all of the injection procedures. Approximately 1 in 5 patients will experience minor transient adverse events with transforaminal or interlaminar epidural steroid injections. No clear estimate of the overall incidence rate for any injection procedure is available due to a lack of reporting. Although we did not find any specific reviews reporting harms associated with oral medication for neck pain, we can be informed by reviews that report adverse events for other musculoskeletal pain disorders for some guidance.

A few systematic reviews of antidepressant side effects have been conducted and Perrot et al. (2008) note that when prescribed for painful conditions, side-effects occur in 30 to $100 \%$ of patients and are often dose-dependent [105]. Reported side effects are dysuria, constipation, dry mouth, drowsiness, eye accommodation disorders, tachycardia, memory disorders and confusion, orthostatic hypotension, dizziness, weight gain, trembling, impotence, nausea, fatigue and serotonergic syndrome. Hauser et al. (2012) provide relative risk estimates for similar mild transient adverse events ranging from $0.94(0.46,1.68)$ for headache to 9.51 $(1.22,74.0)$ for sexual dysfunction [106].

There is a vast literature informing NSAIDS adverse events although the great majority is related to oral and compared to topical use. Zhang et al. report that gastrointestinal events are 3 to 5 times higher compared to placebo, while Jones et al. report that up to $60 \%$ of all NSAID users will experience a GI event [107, 108]. Canadian guidelines for NSAID use reported that the rate of gastrointestinal hemorrhage is 1.5 to $2.0 \%$ per year for average risk patients while Jones et al. report a rate of 1.0 to $1.5 \%$ [108, 109]. The Osteoarthritis Research Society International (OARSI) guidelines reported that cardiovascular events are comparable in the COX-2 class with the traditional NSAIDS blocking both COX-1 and COX-2 (RR 1.19 95\% CI 0.80, 1.75) [107], while Jones et al. argue that selective COX-2 inhibitors and NSAIDs could have different rates [108]. 
Opiates were considered in a recent systematic review of conservative management for low back pain. Opiate use was associated with a greater risk of headache and nausea, drowsiness, dry mouth, constipation, pruritis, vomiting, anorexia and increased sweating over placebo. The risk differential was low and ranged from 3 to 9\% [110]. The OARSI guidelines report a relative risk for any adverse event related to opiate use at 1.4 (95\% CI 1.3, 1.6) and specifies constipation, nausea, drowsiness, dizziness and vomiting as side effects.

A Cochrane review reported that there was no significant difference in the overall safety of Acetaminophen compared to NSAIDS [111]. Acetaminophen can be associated with GI events but at a lower rate than that of NSAIDS. Acetaminophen can lead to renal failure however with a RR up to $2.5(95 \%$ CI $1.7,3.6)[90]$.

\section{CONCLUSION}

There is a lack of trials in neck pain for common injections and medications and this leads to an inability to fuller inform the proper use of pharmacological therapies. The current state of the evidence appears to favor the muscle relaxant eperison hydrochloride for chronic neck pain. Evidence is emerging against IM botulinum toxin-A injections for chronic mechanical neck disorder or subacute/chronic WAD and against medial branch block with steroids for chronic facet joint pain. Given the limited number of trials and the low level of evidence in those that have been performed, coupled with the frequent and disability nature of neck pain, high quality trials of analgesics, anti-inflammatories, and psychotropic agents are urgently needed.

\section{ABBREVIATIONS}

AMSTAR Assessment of the Methodological Quality of Systematic Reviews; CI Confidence Interval; COX Cyclooxygenase; GRADE Grading of Recommendation Assessment, Development, and Evaluation; ICON International Collaboration on Neck; IM intramuscular; IP immediate post; LT long-term; MCID Minimal Clinically Important Difference; MND mechanical neck disorder; NDI Neck Disability Index; NNT Number Needed to Treat; NSAID non-steroidal anti-inflammatory drug; PRISMA Preferred Reporting Items for Systematic Reviews and MetaAnalyses; RCT randomized controlled trial, RR relative risk; $\mathrm{SMD}_{\mathrm{p}}$ Standard Mean Difference (pooled); VAS Visual Analogue Scale; WAD whiplash associated disorder; WMD Weighted Mean Difference.

\section{CONFLICT OF INTEREST}

Paul M. Peloso conducts clinical research for Merck, a company that manufactures and markets classes of drugs named in this review, including NSAIDs and antidepressants. This article represents the views of Dr. Peloso and the ICON team and should not be construed to represent the views of Merck.

\section{ACKNOWLEDGEMENTS}

This work was supported by Canadian Institutes of Health Research (CIHR) grant(s) FRN: KRS-102084 and Centric Health LifeMark Industry Partner Grant.
ICON is a multi-disciplinary collaborative group that includes scientist-authors (listed below) and support staff (Margaret Lomotan) that conduct knowledge synthesis and translation aimed at reducing the burden of neck pain.

The ICON authors that provided direction of the project and reviewed the findings/manuscript include (in alphabetical order): Gert Bronfort, Norm Buckley, Lisa Carlesso, Linda Carroll, Pierre Côté, Jeanette Ezzo, Paulo Ferreira, Tim Flynn, Charlie Goldsmith, Anita Gross, Ted Haines, Jan Hartvigsen, Wayne Hing, Gwendolen Jull, Faith Kaplan, Ron Kaplan, Helge Kasch, Justin Kenardy, Per Kjær, Janet Lowcock, Joy MacDermid, Jordan Miller, Margareta Nordin, Paul Peloso, Jan Pool, Duncan Reid, Sidney Rubinstein, P. Lina Santaguida, Anne Söderlund, Natalie Spearing, Michele Sterling, Grace Szeto, Robert Teasell, Arianne Verhagen, David M. Walton, Marc White.

\section{APPENDIX 1}

\section{Oral Medications and Medical Injections}

\begin{tabular}{|c|c|}
\hline Oral Medication: & Non-Opiate Analgesics \\
\hline \multicolumn{2}{|c|}{ acetaminophen, paracetamol, paramax, migraeflux, metomax } \\
\hline Oral Medication: & Non-steroidal Anti-inflammatory (NSAID) \\
\hline \multicolumn{2}{|c|}{$\begin{array}{l}\text { ibuprofen, naproxen, meloxicam, celecoxib, acetylsalicyl (acetylsalicylic } \\
\text { acid, ASA), carbasalaatcalcium, diflunisal, aceclofenac, alclofenac, } \\
\text { diclofenac, indometacin, sulindac, piroxicam, dexibuprofen, } \\
\text { dexketoprofen, fenoprofen, flurbiprofen, ketoprofen, tiapro (tiaprofenic } \\
\text { acid), metamizol, phenylbutazone, phenazone, propyphenazone, toradol, } \\
\text { etoricoxib, nabumeton (nabumatone), parecoxib, valdecoxib, } \\
\text { lumiracoxib, rofecoxib }\end{array}$} \\
\hline
\end{tabular}

Topical Medication: NSAID

diclofenac, iburofen, diclofenac, salicylic acid, piroxicam, ketoprofen, globus/ menthol/ salicylic acid/ turpentine oil, felbinac, nicotinic acid/ salicylic acid, oleoresin/ iodine/ menthol/ salicylic acid, acetic acid/ turpentine oil, capsicum oleoresin/ nicotinic acid/ salicylic acid, cajuput/camphor/mentha, acetic acid/ammonia/turpentine oil, menthol/ salicylic acid, ammonium/ oleic acid, turpentine oil, camphor/ creosote/ eucalyptus, globules/ menthol/ pinus mugo pumilio/ salicylic acid/ thymus vulgare/ turpentine oil, camphor/ menthol/ salicylic acid, diclofenac/ linum usitatissimum/ menthol/ salicylic acid, camphor/ nicotinic acid/ salicylic acid, benzocaine/ salicylamide, benzocaine/ salicylic acid, benzocaine/ nicotinic acid/ salicylic acid, iodine/ salicylic acid, acetylsalicylic acid/ camphor/menthol/salicylic acid

Oral Medication: Analgesic Opiate/Narcotics

codeine, buprenorphine, tramadol, fentany, hydromonorphone, morphine, oxycodone/ naloxone, opiate, opium, acetyldihydrocodeine, alfentani, allylprodine, alphamethylfentanyl, alphaprodine, benzylmorphine , betaprodine, bezitriamide, buprenorphine, butorphanol, bremazocine, carfentan (carfentanyl), contin, dextromoramide , dextropropoxyphene, dezocine, diacetylmorphine, diamorphine, dihydrocodeine, dihydromorphine, dihydromorphone , diphenoxylate, dipipanone, enadoline, ethylketazocine, ethylmorphine, etonitazene, etorphine, fentanyl, heroin, hydrocodone, hydromorphin (hydromorphine), hydromorphone , ketazocine, ketobemidone, lefetamine, levomethadon, levomethadyl, levomethorphan, levor-phanol, loperamide, meperidine, meptazinol, methadone, methadyl methylmorphine, morphin (morphine), nalbuphine, narcotic, nicocodeine, nicomorphine, normorphine, noscapin, ohmefentanyl, oripavine, oxycodone, oxycontin, oxymorphone, papaveretum, papaverin, pentazocine, percocet, peronine, pethidine, phenazocine, phencyclidine, pholcodine, piritramid (priitramidine), prodine, promedol, propoxyphene, remifentanil, sufentanil, tapentadol, thebaine, tilidine, tramadol, ultracet

Oral Medication: Muscle Relaxants 
baclofen, cyclobenzaprine, eperison hydrochloride, methocarbamol, orphenadrine, tizanidine, chlorzoxazone, metaxalone, meprobamate, zopiclone

Oral Medication: Benzodiazepines

diazepam, alprazolam or xanax or xanor or tafil or alprox or frontal, bromazepam or lexotanil or lexotan or lexomil or somalium or bromam, chlordiazepoxide or librium or tropium or risolid or klopoxid, cinolazepam or gerodorm, clonazepam or klonopin or rivotril or iktorivi, cloxazolam or olcadil, clorazepate or tranxene, diazepam or valium or pax or apzepam or stesolid, estazolam or proSom, flunitrazepam or rohypnol or fluscand or flunipam or rona or rohydorm, flurazepam or dalmadorm or dalmane, flutoprazepam or restas, halazepam or paxipam or ketazolam or anxon or loprazolam or dormonoct, iorazepam or ativan or temesta or tavor, lorabenz, lormetazepam or loramet or noctamid or pronoctan, medazepam or nobrium, midazolam or dormicum or versed or hypnovel or dormonid, nimetazepam or erimin, nitrazepam or mogadon or alodorm or pacisyn or dumolid, nordazepam or madar or stilny, oxazepam or seresta or serax or serenid or serepax or sobril, pinazepam or domar or prazepam or lysanxia or centrax or quazepam or doral, temazepam or restoril or normison or euhypnos or tenox, Tetrazepam or Mylostan or Triazolam or Halcion or Rilamir

\section{Oral Medication: Tricyclic Antidepressants}

amitriptyline (amitriptyline or elavil or endep), desipramine, clomipramine or anafranil, desipramine or norpramin or pertofrane, dosulepin or dothiepin or prothiaden, doxepin or adapin or sinequan, imipramine or tofranil, lofepramine or feprapax or gamanil or lomont, nortriptyline or pamelor, protriptyline or vivactil, trimipramine or surmontil, amoxapine or asendin, loxapine or loxapac or loxitane, maprotiline or deprilept or ludiomil or psymion, mazindol or mazanor or sanorex, mianserin or bolvidon or norval or tolvon, mirtazapine or remeron or avanza or zispin, setiptiline or tecipul

\begin{tabular}{l}
\hline Oral Medication: GABA Derivatives \\
\hline gabapentin, pregabalin \\
\hline Medical Injections: Corticosteroids \\
\hline $\begin{array}{l}\text { betametson, methylprednisolone, triamcinolone acetomide, } \\
\text { triamcinolone, steroid of corticosteroid, prednisone, prednisolone, } \\
\text { betamethasone }\end{array}$
\end{tabular}

Medical Injections: Analgesics

procaine, lidocaine, prilocaine, benzocaine, bupiviciane, mepivacine, articaine, tetracaine, ropivacaine, lignocaine, mexiletine, flecainide, tocainide

Medical Injections: Neuromuscular Blocking Agent

Botulinum toxin Type A, botulinum toxin type B

\section{APPENDIX 2}

\section{Achieving Clinically Meaningful Comparisons Between Studies}

Treatment efficacy outcomes of primary interest and most commonly reported were pain intensity (e.g., Visual Analog Scale-VAS, NRS, McGill Pain Questionnaire-MPQ) and disability (e.g., Neck Disability Index - NDI, Northwick Park Neck Pain Questionnaire-NPQ, Pain Disability IndexPDI). The magnitude of effect can be estimated for continuous outcomes - the effect size (SMD; WMD) and for binary outcomes (i.e. yes, no) - NNT to achieve this effect. The degree of clinical importance for the observed differences in pain scores between the treatment groups was specified according to the Updated Method Guidelines of Cochrane Collaboration Back Review Group [12] and tradition effect size (Cohen d) [13] estimation.

\begin{tabular}{|c|c|c|c|c|}
\hline $\begin{array}{l}\text { Clinical } \\
\text { Importance }\end{array}$ & $\begin{array}{l}\text { Pain } \\
\text { Intensity }\end{array}$ & $\begin{array}{l}\text { Function (Self } \\
\text { Report) }\end{array}$ & $\begin{array}{l}\text { Effect } \\
\text { Size } \\
\text { Cohen d } \\
\text { (SMD) }\end{array}$ & GPE \\
\hline $\begin{array}{l}\text { Small } \\
\text { (A little better) }\end{array}$ & $\begin{array}{l}\text { WMD }< \\
10 \% \text { of the } \\
\text { VAS scale }\end{array}$ & $\begin{array}{l}\text { Neck Disability } \\
\text { Index (NDI) } \\
\text { MDC 5/50 for } \\
\text { uncomplicated } \\
\text { neck pain; up to } \\
10 / 50 \text { for } \\
\text { radiculopathy; } \\
\text { Clinically } \\
\text { important } \\
\text { difference varies } \\
\text { across studies } \\
\text { from 5/50 to } \\
\text { 19/50 [14] } \\
\text { Northwick Park } \\
\text { Neck Pain } \\
\text { Questionnaire } \\
\text { (NPQ) unclear } \\
\text { Pain Disability } \\
\text { Index (PDI) } \\
\text { unclear }\end{array}$ & $\begin{array}{l}0.2 \text { as } \\
\text { small }\end{array}$ & $\begin{array}{l}\text { little } \\
\text { improvem } \\
\text { ent } \\
\text { MID }\end{array}$ \\
\hline $\begin{array}{l}\text { Medium } \\
\text { (Somewhat better) }\end{array}$ & \begin{tabular}{|l}
$10 \% \leq$ \\
WMD $<$ \\
$20 \%$ of the \\
VAS scale \\
neuropathic \\
pain at least \\
$30 \%$ pain \\
reduction \\
from \\
baseline \\
no worse \\
than mild \\
pain \\
remaining \\
{$[15]$}
\end{tabular} & $\begin{array}{l}15 \% \text { rule from } \\
\text { Ottawa Panel - } \\
\text { rheumatologists } \\
\text { survey } \\
\text { WMD } \geq 10 \text { NDI } \\
\text { units }\end{array}$ & $\begin{array}{l}\geq 0.5 \text { as } \\
\text { medium }\end{array}$ & $\begin{array}{l}\text { moderate } \\
\text { improvem } \\
\text { ent }\end{array}$ \\
\hline $\begin{array}{l}\text { Large } \\
\text { (A lot better) }\end{array}$ & $\begin{array}{l}\text { WMD } \geq \\
20 \% \text { of the } \\
\text { VAS scale } \\
\text { neuropathic } \\
\text { pain at least } \\
50 \% \\
\text { reduction } \\
\text { from } \\
\text { baseline; } 50 \\
\text { to } 70 \% \text { is a } \\
\text { clinical } \\
\text { success [15] } \\
\text { Final pain } \\
\text { intensity < } \\
30 / 100 m m \\
\text { or equivalent } \\
\text { State: No } \\
\text { worse than } \\
\text { mild pain. }\end{array}$ & & $\begin{array}{l}\geq 0.8 \text { as } \\
\text { large }\end{array}$ & $\begin{array}{l}\text { a lot of } \\
\text { improvem } \\
\text { ent }\end{array}$ \\
\hline
\end{tabular}

Key: WMD - weighted mean difference, VAS - visual analogue scale, NDI - neck disability index, GPE - global perceived effect

\section{APPENDIX 3}

Excluded studies are listed for medical injections and oral medications with reason for exclusion in square brackets.

Excluded for Medicinal Injections 
Lee M, Choi T, Kim J, Choi S. Using Guasha to treat musculoskeletal pain: A systematic review of controlled clinical trials. Chin Med 2010; 5: 5. [Intervention]

Excluded for Oral Medications

Belachew DA, Schaller BJ, Guta Z. Cervical spondylosis: a literature review with attention to the African population. Arch Med Sci 2007; 3(4): 315-22. [Intervention]

Bronfort G, Nilsson N, Haas M, et al. Non-invasive physical treatments for chronic/recurrent headache. Cochrane Database Syst Rev 2004; (3): CD001878. [Comparison]

Conlin A, Bhogal S, Sequeira K, Teasell R. Treatment of whiplashassociated disorders--part I: Non-invasive interventions. Pain Res Manag 2005 Spring; 10(1): 21-32. [Comparison]

Drescher K, Hardy S, MacLean J, Schindler M, Scott K, Harris SR. Efficacy of postural and neck-stabilization exercises for persons with acute whiplash-associated disorders: a systematic review. Physiother Can 2008; 60(3): 215-23. [Comparison]

Kay TM, Gross A, Goldsmith C, et al. Exercises for mechanical neck disorders. Cochrane Database Syst Rev 2005; 3: CD004250. [Comparison]

Kroeling P, Gross A, Goldsmith CH, et al. Electrotherapy for neck pain (Review). Electrotherapy for neck pain. Cochrane Database Syst Rev 2009; 4: CD004251. [Outcome]

Leininger B, Bronfort G, Evans R, Reiter T. Spinal manipulation or mobilization for radiculopathy: a systematic review. Phys Med Rehabil Clin N Am 2011; 22(1): 105-25. [Comparison]

Miller J, Gross A, D'Sylva J, et al. Manual therapy and exercise for neck pain: a systematic review. Man Ther 2010; 15(4): 334-54. [Intervention] Nikolaidis I, Fouyas IP, Sandercock PA, Statham PF. Surgery for cervical radiculopathy or myelopathy. Cochrane Database Syst Rev 2010; 1: CD001466. [Outcome]

Reid SA, Rivett DA. Manual therapy treatment of cervicogenic dizziness: a systematic review. Man Ther 2005; 10(1): 4-13. [Outcome] Salt E, Wright C, Kelly S, Dean A. A systematic literature review on the effectiveness of non-invasive therapy for cervicobrachial pain. Man Ther. 2011; 16(1): 53-65. [Intervention]

Teasell RW, McClure JA, Walton D, et al. A research synthesis of therapeutic interventions for whiplash-associated disorder (WAD): Part 2 - interventions for acute WAD. Pain Res Manage 2010; 15(5): 295304. [Comparison]

Trinh K, Graham N, Gross A, et al. Acupuncture for neck disorders. Spine 2007; 32(2): 236-43. [Intervention]

Verhagen AP, Scholten-Peeters GG, de Bie RA, Bierma-Zeinstra SM. Conservative treatments for whiplash. Cochrane Database Syst Rev 2007; 2: CD003338. [Intervention]

Vernon HT, Humphreys BK, Hagino CA. A systematic review of conservative treatments for acute neck pain not due to whiplash. $\mathrm{J}$ Manipulative Physiol Ther 2005; 28(6): 443-8. [Comparison]

Vernon H, Humphreys BK. Manual therapy for neck pain: an overview of randomized clinical trials and systematic reviews. Eura Medicophys 2007; 43(1): 91-118. [Comparison]

\section{REFERENCES}

[1] Croft PR, Lewis M, Papageorgiou AC, et al. Risk factors for neck pain: a longitudinal study in the general population. Pain 2001; 93(3): 317-25.

[2] Hogg-Johnson S, van der Velde G, Carroll LJ, et al. The burden and determinants of neck pain in the general population: results of the Bone and Joint Decade 2000-2010 Task Force on Neck Pain and Its Associated Disorders. Spine 2008; 33(4S): S39-51.

[3] Chevan J, Riddel DL. Factors Associated With Care Seeking From Physicians, Physical Therapists, or Chiropractors by Persons With Spinal Pain: A Population-Based Study. J Orthop Sports Phys Ther 2011; 42(7): 467-76.

[4] Chou R, McDonagh M, Nakamoto E, et al. Comparative Effectiveness and Safety of Analgesics for Osteoarthritis: An Update of the 2006 Report. Comparative Effectiveness Review No. 38 (Prepared by Oregon Evidence-based Practice Center under Contract No. HHSA 290-2007-10057-I). Rockville, MD: Agency for Healthcare Research and Quality; October 2011. AHRQ Publication No. 11(12)-EHC076EF. Available at: http://www.effectivehealthcare.ahrq.gov/reports/analg esicsupdate.cfm.
[5] Canadian Guidelines for Safe and Effective Use of Opioids for Chronic Non-Cancer Pain. April 30 2010. National Opioid Use Guideline Group. Available at: http://www.nationalpaincentre.mcmaster.ca/opioid Gilron I, Bailey JM, Tu D, et al. Morphine, gabapentin, or their combination for neuropathic pain. N Engl J Med 2005; 352: 1324-34.

[7] Peloso P, Gross A, Haines T, et al. Medicinal and injection therapies for mechanical neck disorders (Review). Cochrane Database Syst Rev 2007; (3): CD000319.

[8] Shea BJ, Hamel C, Wells GA, et al. AMSTAR is a reliable and valid measurment tool to assess the methodological quality of systematic reviews. J Clin Epidemiol 2009; 62(10): 1013-20.

[9] Balshem H, Helfand M, Schunemann HJ, et al. GRADE guidelines: 3. Rating the quality of evidence. J Clin Epidemiol 2011; 64(4): 401-6.

[10] Santaguida L, MacDermid J, Gross A, et al. ICON Methodology. Orthopaed Online J 2013, (accepted).

[11] Whitlock EP, Lin JS, Chou R, Shekelle P, Robinson KA. Using existing systematic reviews in complex systematic reviews. Ann Intern Med 2008; 148: 776-82.

[12] Furlan AD, Pennick V, Bombardier C, van TM. 2009 updated method guidelines for systematic reviews in the Cochrane Back Review Group. Spine (Phila Pa 1976). 2009; 34: 1929-41.

[13] Cohen J. Statistical power analysis for the behavioural sciences. Hillside NJ: Lawrence Erlbaum Associates 1988.

[14] MacDermid JC, Walton DM, Avery S, et al. Measurement properties of the neck disability index: a systematic review. J Orthop Sports Phys Ther 2009; 39(5): 400-17.

[15] Cillins SL, Moore RA, McQuay HJ. The visual analogue pain intensity scale: What is moderate pain in millimetres? Pain 1997; 72: 95-7.

[16] Moher D, Liberati A, Tetzlaff J, Altman DG, The PRISMA Group. Preferred Reporting Items for Systematic Reviews and Meta-Analyses: The PRISMA Statement. PLoS Med 2009; 6(6): e1000097.

[17] Pettersson K, Toolanen G. High-dose methylprednisolone prevents extensive sick leave after whiplash injury. Spine 1998; 23: 984-9.

[18] Conlin A, Bhogal S, Sequeira K, Teasell R. Treatment of whiplashassociated disorders - Part II: Medical and surgical interventions. Pain Res Manag 2005; 10(1): 33-40.

[19] Esenyel M, Caglar N, Aldemir T. Treatment of myofascial pain. Am J Phys Med Rehabil 2000; 79(1): 48-52.

[20] Gross AR, Goldsmith CH, Hoving JL et al. Conservative management of mechanical neck disorders: a systematic review. J Rheumatol 2007; 34(5): 1083-102.

[21] Hong CZ. Lidocaine injection versus dry needling to myofascial trigger point: the importance of the local twitch response. Am J Phys Med Rehabil 1994; 73(4): 256-63.

[22] Tsakitzidis G, Remmen R, Peremans L, et al. Non-specific neck pain: diagnosis and treatment. Good Clinical Practice (GCP). KCE Reports 119C. D/2009/10.273/56.Brussels: Belgian Health Care Knowledge Centre (KCE). 2009.

[23] Braker C, Yariv S, Adler R, Badarny S, Eisenberg E. The analgesic effect of botulinum-toxin A on postwhiplash neck pain. Clin J Pain 2008; 24: 5-10.

[24] Lew HL, Lee EH, Castaneda A, Klima R, Date E. Therapeutic use of botulinum toxin type $\mathrm{A}$ in treating neck and upper-back pain of myofascial origin: a pilot study. Arch Phys Med Rehabil 2008; 89: 7580.

[25] Ferrante FM, Bearn L, Rothrock R, King L. Evidence against trigger point injection technique for the treatment of cervicothoracic myofascial pain with botulinum toxin type A. Anesthesiology 2005; 103: 377-83.

[26] Langevin P, Lowcock J, Weber J, et al. Botulinum Toxin Intramuscular Injections for Neck Pain: A Systematic Review and Metaanalysis. J Rheumatol 2011; 38: 203-14.

[27] Stav A, Ovadia L, Sternberg A, Kaadan M, Weksler N. Cervical epidural steroid injection for cervicobrachialgia. Acta Anaesthesiol Scand 1993; 37: 562-6.

[28] Castagnera L, Maurette P, Pointillart V, Vital JM, Erny P, Senegas J. Long term results of cervical epidural steroid injection with and without morphine in chronic cervical radicular pain. Pain 1994; 58: 239-43.

[29] Pasqualucci A, Varrassi G, Braschi A, et al. Epidural local anesthetic plus corticosteroid for the treatment of cervical brachial radicular pain: Single injection versus continuous infusion. Clin J Pain 2007; 23: 5517.

[30] Benyamin RM, Singh V, Parr AT, Conn A, Diwan S, Abdi S. Systematic review of the effectiveness of cervical epidurals in the management of chronic neck pain. Pain Physician 2009; 12(1): 137-57. 
[31] Abdi S, Datta S, Trescot AM, et al. Epidural steroids in the management of chronic spinal pain: a systematic review. Pain Physician 2007; 10(1): 185-212.

[32] Abdi S, Datta S, Lucas LF. Role of epidural steroids in the management of chronic spinal pain: a systematic review of effectiveness and complications. Pain Physician 2005; 8(1): 127-43.

[33] Manchikanti L, Damron K, Cash K, Manchukonda R, Pampati V. Therapeutic cervical medial branch blocks in managing chronic neck pain: a preliminary report of a randomized, double-blind, controlled trial. Clinical trial Nct0033272. Pain Physician 2006; 9(4): 333-46.

[34] Manchikanti L, Manchikanti K, Pampati V, Brandon D, Giordano J. The prevalence of facet joint-related chronic neck pain in postsurgical and non-postsurgical patients: A comparative evaluation. Pain Pract 2008; 8: 5-10.

[35] Boswell MV, Colson JD, Sehgal N, Dunbar EE, Epter R. A systematic review of therapeutic facet joint interventions in chronic spinal pain. Pain Physician 2007; 10(1): 229-53.

[36] Falco FJ, Erhart S, Wargo BW, et al. Systematic review of diagnostic utility and therapeutic effectiveness of cervical facet joint interventions. Pain Physician 2009; 12(2): 323-44.

[37] Byrn C, Olsson I, Falkheden L, et al. Subcutaneous sterile water injections for chronic neck and shoulder pain following whiplash injuries. Lancet 1993; 341: 449-52.

[38] Teasell RW, McClure JA, Walton D, et al. A research synthesis of therapeutic interventions for whiplash-associated disorder (WAD): Part 4 - noninvasive interventions for chronic WAD. Pain Res Manag 2010; 15(5): 313-22.

[39] Teasell RW, McClure JA, Walton D, et al. A research synthesis of therapeutic interventions for whiplash-associated disorder (WAD): Part 5 - surgical and injection-based interventions for chronic WAD. Pain Res Manag 2010; 15(5): 323-34.

[40] Nasswetter G, de los Santos AR, Marti ML, Girolamo GD. [Asociacion de Clonixinato de Lisina con Ciclobenzaprina en afecciones dolorosas del raquis con contractura muscular.] Lysine Clonixinate Association with Cyclobenzaprine in painful conditions of the spine with muscle spasm. Prensa Med Argent 1998; 85: 507-14.

[41] Salzmann VE, Wiedemann O, Loffler L, Sperber H. [Tetrazepam in der behandlung akuter zervikalsyndrome, randomisierte dopple-blinde pilotstudie zum vergleich von Tetraepam und plazebo.]

Tetrazepam in the treatment of acute zervikalsyndrome, dopple-blind randomized pilot study for comparison of Tetraepam and placebo. Fortschr Med 1993; 34: 544-8.

[42] Bose K. The efficacy and safety of eperisone in patients with cervical spondylosis: results of a randomized, double-blind, placebo-controlled trial. Methods Find Exp Clin Pharmacol 1999; 21(3): 209-13.

[43] Berry H, Liyanage SP, Durance RA, et al. A double-blind study of benorylate and chlormezanone in musculoskeletal disease. Rheumatol Rehabil 1981; 20: 46-9.

[44] Hurwitz EL, Carragee EJ, van der Velde G, et al. Bone and Joint Decade 2000-2010 Task Force on Neck Pain and Its Associated Disorders (Review). Spine 2008; 33(4S): S123-52.

[45] Yamamoto M, Sugano T, Kashiwazaki S, et al. Double-blind comparison of piroxicam and indomethacin in the treatment of cervicobrachial syndrome and periarthritis scapulohumeralis (stiff shoulder). Eur J Rheumatol Inflamm 1983; 6: 266-73.

[46] Ginsbert F, Famaey JP. Double-blind study of tolmetin $v s$ naproxen in the treatment of cervical and lumbar osteoarthritis. Curr Ther Res 1980; 26(5): 622-9.

[47] Hoivik HO, Moe N. Effect of a combination of orphenadrine/paracetamol tablets ('Norgesic') on myalgia: a double-blind comparison with placebo in general practice. Curr Med Res Opin 1983; 8: 531-5.

[48] Leaver AM, Refshauge KM, Maher CG, McAuley JH. Conservative interventions provide short-term relief for non-specific neck pain: a systematic review. J Physiother 2010; 56: 73-85.

[49] Ma K, Jiang W, Zhou Q, Du DP. The efficacy of oxycodone for management of acute pain episodes in chronic neck pain patients. Int $\mathbf{J}$ Clin Pract 2008; 62(2): 241-7.

[50] Cheshire WP, Abashian SW, Mann JD. Botulinum toxin in the treatment of myofascial pain syndrome. Pain 1994; 59: 65-9.

[51] Gobel H, Heinze A, Reichel G, Hefter H, Benecke R. Efficacy and safety of a single botulinum type A toxin complex treatment (Dysport) for the relief of upper back myofascial pain syndrome: Results from a randomized double-blind placebo-controlled multicentre study. Pain 2006; 125: 82-8.

[52] Ojala T, Arokoski JP, Partanen J. The effect of small doses of botulinum toxin $\mathrm{A}$ on neck-shoulder myofascial pain syndrome: a double-blind, randomized, and controlled crossover trial. Clin J Pain 2006; 22: 90-6.

[53] Schnider P, Moraru E, Vigl M, et al. Physical therapy and adjunctive botulinum toxin type $\mathrm{A}$ in the treatment of cervical headache: a doubleblind, randomised placebo controlled study. J Headache Pain 2002; 3: 93-9.

[54] Freund BJ, Schwartz M. Treatment of chronic cervical-associated headache with Botulinum Toxin-A: A pilot study. Headache 2000; 40: 231-6.

[55] Wheeler AH, Goolkasian P, Gretz SS. Botulinum toxin A for the treatment of chronic neck pain. Pain 2001; 94: 255- 60.

[56] Carroll A, Barnes M, Comiskey C. A prospective randomized controlled study of the role of botulinum toxin in whiplash-associated disorder. Clin Rehabil 2008; 22: 513-9.

[57] Padberg M, de Bruijn SF, Tavy DL. Neck pain in chronic whiplash syndrome treated with botulinum toxin. A double-blind, placebocontrolled clinical trial. J Neurol 2007; 254: 290-5.

[58] Freund BJ, Schwartz M. Treatment of whiplash associated neck pain with botulinum toxin-A: a pilot study. J Rheumatol 2000; 27: 481-4.

[59] Dennert VR, Munzenberg KJ, Haase W. [Zur therapie der ZervikoBrachialgie, Controllierter klinischer vergleich einer hochdosierten kombination neurotroper Vitamine mit einem Analgetikum.] For therapy of Cervico-brachialgia, control profiled comparative clinical high-dose combination of neurotropic vitamins with an analgesic. Fortschr Med 1976; 94(10): 595-8.

[60] Barnsley L, Lord SM, Wallis BJ, et al. Lack of effect of intraarticular corticosteroids for chronic pain in the cervical zygapophyseal joints. New Engl J Med 1994; 330: 1047-50.

[61] Carragee EJ, Hurwitz EL, Cheng I, et al. Treatment of neck pain: injections and surgical interventions: results of the Bone and Joint Decade 2000-2010 Task Force on Neck Pain and Its Associated Disorders. Spine 2008; 33(4S): S153-69.

[62] Sand T, Bovim G, Held G. Intracutaneous sterile water injections do not relieve pain in cervicogenic headache. Acta Neurol Scand 1992; 86: 526-8.

[63] Brockow T, Dillner A, Franke A, Resch KL. Analgesic effectiveness of subcutaneous carbon-dioxide insufflations as an adjunct treatment in patients with non-specific neck or low back pain. Complement Ther Med 2001; 9: 68-76.

[64] Thomas M, Eriksson SV, Lundeberg T. A comparative study of diazepam and acupuncture in patients with osteoarthritis pain: a placebo controlled study. Am J Chin Med 1991; 19(2): 95-100.

[65] Basmajian JV. Cyclobenzaprine Hydrochloride Effect on Skeletal Muscle Spasm in the Lumbar Region and Neck: Two Double-Blind Controlled Clinical and Laboratory Studies. Arch Phys Med Rehabil 1978; 59(2): 58-63.

[66] Basmajian JV. Reflex cervical muscle spasm: Treatment by Diazepam, Phenobarbital or placebo. Arch Phys Med Rehabil 1983; 64: 121-4.

[67] Sloop PR, Smith DS, Goldenberg E, Dore C. Manipulation for chronic neck pain. A double-blind controlled study. Spine 1982; 7(6): 532-5.

[68] Furlan A, Yazdi F, Tsertsvadze A, et al. Complementary and Alternative Therapies for Back Pain II. Evidence Report/Technology Assessment No. 194. (Prepared by the University of Ottawa Evidencebased Practice Center under Contract No. 290-2007-10059-I (EPCIII). AHRQ Publication No. 10(11)E007. Rockville, MD: Agency for Healthcare Research and Quality. October 2010.

[69] Gross A, Miller J, D’Sylva J, et al. Manipulation or Mobilisation for Neck Pain. Cochrane Database Syst Rev 2010; 1: CD004249.

[70] Furtado RNV, Carazzato S, Farias CA, Chamlian TR, Masiero D. Myofascial syndrome: comparison between infiltration of trigger points treatment and oral medication (cyclobenzaprine) [Síndrome miofascial: comparação entre o tratamento com infiltração de trigger points e medicação oral (ciclobenzaprina)]. Acta Fisiátrica 2002; 9(3): 117-26.

[71] Leite FM, Atallah AN, El Dib R, et al. Cyclobenzaprine for the treatment of myofascial pain in adults. Cochrane Database Syst Rev 2009; 3: CD006830.

[72] Payne RW, Sorenson EJ, Smalley TK, Brandt EN. Diazepam, meprobamate and placebo in musculoskeletal disorders. JAMA 1964; 188(3): 157-60.

[73] Schreiber S, Svetiana V, Shavelzon V, Pick CG, Zahavi E, Shir Y. A randomized trial of fluoxetine versus amitriptyline in musculoskeletal pain. Isr J Psychiatry Relat Sci 2001; 38(2): 88-94.

[74] Giles LG, Muller R. Chronic spinal pain: a randomized clinical trial comparing medication, acupuncture, and spinal manipulation. Spine 2003; 28(14): 1490-502. 
[75] Giles LG, Muller R. Chronic spinal pain syndromes: A clinical pilot trial comparing acupuncture, a non-steroidal anti-inflammatory drug, and spinal manipulation. J Manipulative Physiol Ther 1999; 22(6): 37681.

[76] Furlan AD, Yazdi R, Tsertsvadze A, et al. A systematic review and meta-analysis of efficacy, cost-effectiveness, and safety of selected complementary and alternative medicine for neck and low-back pain. Evid Based Complement Alternat Med 2012; 2012: 953139.

[77] Koes BW, Bouter LM, van Mameren H, et al. The effectiveness of manual therapy, physiotherapy, and treatment by the general practitioner for nonspecific back and neck complaints. Spine 1992c; 17(1): 28-35.

[78] Haraldsson B, Gross A, Myers CD, et al. Massage for mechanical neck disorders. Cochrane Database Syst Rev 2006; 3: CD004871.

[79] Muller R, Giles LG. Long-term follow-up of a randomized clinical trial assessing the efficacy of medication, acupuncture, and spinal manipulation for chronic mechanical spinal pain syndromes. J Manipulative Physiol Ther 2005; 28: 3-11.

[80] Vernon H, Humphreys K, Hagino C. Chronic mechanical neck pain in adults treated by manual therapy: a systematic review of change scores in randomized clinical trials. J Manipulative Physiol Ther 2007; 30: 215-27.

[81] Dostal C, Pavelka K, Lewit K. Ibuprofen v lecbe cervickokranial-niho syndromu v kombinaci manipulacni lecbou. Fysiatr Vestn 1978; 56(5): 258-63.

[82] Birch S, Jamison RN. Controlled trial of Japanese acupuncture for chronic myofascial neck pain: assessment of specific and nonspecific effects of treatment. Clin J Pain 1998; 14: 248-55.

[83] Vernon H, Schneider M. Chiropractic management of myofascial trigger points and myofascial pain syndrome: a systematic review of the literature. J Manipulative Physiol Ther 2009; 32: 14-24.

[84] Shakoor MA, Ahmed MS, Kibria G, et al. Effects of cervical traction and exercise therapy in cervical spondylosis. Bangladesh Med Res Counc Bull 2002; 28: 61-9.

[85] Graham N, Gross AR, Goldsmith C, Cervical Overview Group. Mechanical Traction for mechanical neck disorders: A systematic review. J Rehabil Med 2006; 38: 145-52.

[86] Peake N, Harte A. The effectiveness of cervical traction. Phys Ther Rev 2005; 10: 217-29.

[87] Fu LM, Li JT, Wu WS. Randomized Controlled Trials of acupuncture for neck pain: Systematic review and meta-analysis. J Altern Complement Med 2009; 15(2): 133-45.

[88] Choffray DU, Crielaard JM, Albert A, Franchimont P. Comparative study of high bio-availability glaphenine and paracetamol in cervical and lumbar arthrosis. Clin Rheumatol 1987; 6(4): 518-25.

[89] van Wieringen S, Jansen T, Smits MG, Nagtegaal JF, Coenen AML. Melatonin for chronic whiplash syndrome with delayed melatonin onset - randomized, placebo-controlled trial. Clin Drug Investig 2001; 21(12): 813-20.

[90] Langevin P, Peloso PMJ, Lowcock J, et al. Botulinum toxin for subacute/chronic neck pain (Review). Cochrane Database Syst Rev 2011; 7: CD008626.

[91] Terzi T, Karakurum B, Ucler S, Inan LE, Tulumay C. Greater occipital nerve blockade in migraine, tension-type headache and cervicogenic headache. J Headache Pain 2002; 3: 137-41.

[92] Inan N, Ceyhan A, Inan L, Kavaklioglu O, Alptekin A, Unal N. C2/C3 Nerve blocks and greater occipital nerve block in cervicogenic headache treatment. Funct Neurol 2001; 16(3): 239-43.

[93] Malhotra G, Abbasi A, Rhee M. Complications of transforaminal cervical epidural steroid injections. Spine (Phila Pa 1976) 2009; 34 : 731-9.
[94] Guzman J, Haldeman S, Carroll LJ, et al. Clinical practice implications of the Bone and Joint Decade 2000-2010 Task Force on Neck Pain and Its Associated Disorders: From concepts and findings to recommendations. Spine (Phila Pa 1976) 2008; 33: S199-213.

[95] Abdi S, Datta S, Lucas LF. Role of epidural steroids in the management of chronic spinal pain: a systematic review of effectiveness and complications. Pain Physician 2005; 8: 127-43.

[96] Abbasi A, Malhotra G, Malanga G, et al. Complications of interlaminar cervical epidural steroid injections: a review of the literature. Spine (Phila Pa 1976) 2007; 32: 2144-51.

[97] Higa K, Hirata K, Hirota K, et al. Retropharyngeal hematoma after stellate ganglion block: Analysis of 27 patients reported in the literature. Anesthesiology 2006; 105: 1238-45; discussion 5A-6A.

[98] Boswell MV, Colson JD, Spillane WF. Therapeutic facet joint interventions in chronic spinal pain: A systematic review of effectiveness and complications. Pain Physician 2005; 8: 101-14.

[99] Huston CW. Cervical epidural steroid injections in the management of cervical radiculitis: interlaminar versus transforaminal. A review. Curr Rev Musculoskelet Med 2009; 2: 30-42.

[100] van Tulder MW, Touray T, Furlan AD, Solway S, Bouter LM. Muscle relaxants for non-specific low-back pain. Cochrane Database Syst Rev 2003; 2: CD004252.

[101] MacDermid J, Walton D, Miller J, ICON. What is the experience of receiving healthcare for neck pain? Open Orthopaed J 2013; 7: (Suppl 4): M5.

[102] Aker P, Gross AR, Goldsmith C, Peloso P. Conservative management of mechanical neck pain: systematic overview and meta-analysis. BMJ 1996; 313(7068): 1291-6.

[103] Goldsmith CH, Gross AR, MacDermid J, Santaguida L, Miller J. What does the evidence tell us about design of future treatment trials for whiplash-associated disorders? Spine 2011; 36(25S): S292-302.

[104] Schulz KF, Altman DG, Moher D for the CONSORT Group. CONSORT 2010. Statement: Updated guidelines for reporting parallel group randomized trials. Ann Intern Med 2010: 152(11): 1-7.

[105] Perrot S, Javier RM, Marty M, Le Jeunne C, Laroche F, Cedr FRSPSS. Is there any evidence to support the use of anti-depressants in painful rheumatological conditions? Systematic review of pharmacological and clinical studies. Rheumatology (Oxford) 2008; 47: 1117-23.

[106] Hauser W, Wolfe F, Tolle T, Uceyler N, Sommer C. The role of antidepressants in the management of fibromyalgia syndrome: a systematic review and meta-analysis. CNS Drugs 2012; 26: 297-307.

[107] Zhang W, Moskowitz RW, Nuki G, et al. OARSI recommendations for the management of hip and knee osteoarthritis, part I: critical appraisal of existing treatment guidelines and systematic review of current research evidence. Osteoarthr Cartil 2007; 15: 981-1000.

[108] Jones R, Rubin G, Berenbaum F, Scheiman J. Gastrointestinal and cardiovascular risks of nonsteroidal anti-inflammatory drugs. Am J Med 2008; 121: 464-74.

[109] Rostum A, Moayyedi P, Hunt R. Canadian consensus guidelines on long-term nonsteroidal anti-inflammatory drug therapy and the need for gastroprotection: benefits versus risks. Aliment Pharmacol Ther 2009; 29: 481-96.

[110] White AP, Arnold PM, Norvell DC, Ecker E, Fehlings MG. Pharmacologic management of chronic low back pain: synthesis of the evidence. Spine (Phila Pa 1976) 2011; 36: S131-43.

[111] Towheed TE, Maxwell L, Judd MG, Catton M, Hochberg MC, Wells G. Acetaminophen for osteoarthritis. Cochrane Database Syst Rev 2006; (1): CD004257. 